\title{
1 Dynamic evolution of small signaling peptide compensation in plant 2 stem cell control
}

4 Choon-Tak Kwon ${ }^{1,2}$, Lingli Tang ${ }^{3,4}$, Xingang Wang ${ }^{1}$, Iacopo Gentile ${ }^{1}$, Anat Hendelman ${ }^{1}$, Gina

5 Robitaille ${ }^{1,7}$, Joyce Van Eck ${ }^{5,6}, \mathrm{Cao} \mathrm{Xu}^{3,4^{*}}$, Zachary B. Lippman ${ }^{1,7, *}$

$7 \quad{ }^{1}$ School of Biological Sciences, Cold Spring Harbor Laboratory, Cold Spring Harbor, New York 811724, USA.

$9{ }^{2}$ Department of Horticultural Biotechnology, Kyung Hee University, Yongin 17104, Republic of 10 Korea

$11{ }^{3}$ State Key Laboratory of Plant Genomics, National Center for Plant Gene Research, CAS-JIC 12 Centre of Excellence for Plant and Microbial Science (CEPAMS), Institute of Genetics and 13 Developmental Biology, Chinese Academy of Sciences, Beijing, China.

$14{ }^{4}$ University of Chinese Academy of Sciences, Beijing, China.

$15{ }^{5}$ Boyce Thompson Institute, Ithaca, NY 14853, USA

$16{ }^{6}$ Plant Breeding and Genetics Section, School of Integrative Plant Science, Cornell University, 17 Ithaca, NY 14853, USA

$18{ }^{7}$ Howard Hughes Medical Institute, Cold Spring Harbor Laboratory, Cold Spring Harbor, NY 1911724, USA

20

$21 *$ e-mail: caoxu@genetics.ac.cn, lippman@cshl.edu

22

23 Keywords: Paralogs, Redundancy, Epistasis, Signaling Peptide, cis-regulatory, Meristem, Tomato, 24 Solanaceae, CRISPR 
SUMMARY

Gene duplications are a hallmark of plant genome evolution and a foundation for genetic interactions that shape phenotypic diversity ${ }^{1-5}$. Compensation is a major form of paralog interaction ${ }^{6-8}$, but how compensation relationships change as allelic variation accumulates is unknown. Here, we leveraged genomics and genome editing across the Solanaceae family to capture the evolution of compensating paralogs. Mutations in the stem cell regulator $C L V 3$ cause floral organs to overproliferate in many plants ${ }^{9-11}$. In tomato, this phenotype is partially suppressed by transcriptional upregulation of a closely related $\operatorname{paralog}^{12}$. Tobacco lost this paralog, resulting in no compensation and extreme clv3 phenotypes. Strikingly, the paralogs of petunia and groundcherry nearly completely suppress $c l v 3$, indicating a potent ancestral state of compensation. Cross-species transgenic complementation analyses show this potent compensation partially degenerated in tomato due to a single amino acid change in the paralog and cis-regulatory variation that limits its transcriptional upregulation. Our findings show how genetic interactions are remodeled following duplications, and suggest that dynamic paralog evolution is widespread over short time scales and impacts phenotypic variation from natural and engineered mutations.

Gene duplications arise from whole genome and small-scale duplications and are pervasive in plant genomes ${ }^{3,5,13,14}$. Paralogs that emerge from duplications are completely redundant, which allows genetic variation to accumulate under relaxed selection ${ }^{3,5}$. This mutational drift can diversify paralog relationships through gene loss (pseudogenization), partitioning of ancestral functions (subfunctionalization), or gain of novel functions (neofunctionalization) ${ }^{1,3,5,15}$. Another prominent but less understood path of paralog evolution leads to "active compensation", a form of redundancy where one or more paralogs are transcriptionally upregulated to substitute for the compromised activity of another $6,16,17$. Such relationships provide robustness against genetic or environmental change and may be under selection ${ }^{18,19}$. However, an often underappreciated paradox is that while duplications initially provide redundancy, they also promote new genetic

51 variation through relaxed purifying selection ${ }^{18,20,21}$. Such variation, which can accumulate across

52 both coding and cis-regulatory sequences, is the foundation for the broadly studied end-points of

53 paralog diversification. What remains unclear is how such diversification modifies paralog 54 functional relationships as species diversify over shorter time frames. This is because functional 
divergent species, and thus have failed to capture the trajectories and functional consequences of evolving compensatory relationships following lineage-specific ancestral duplications ${ }^{6,12,14}$. important gene family in plants encoding small-signaling peptides with diverse roles in growth and development ${ }^{22,23}$. CLE peptides are 12- or 13-residue glycopeptides processed from prepropeptides $^{23,24}$. The number and functional relationships, including redundancy, of CLE family members, vary considerably between distantly related species, due to lineage-specific duplications and variation in paralog retention and diversification ${ }^{22}$. However, the founding member from Arabidopsis thaliana (arabidopsis), CLAVATA3 (CLV3), is deeply conserved ${ }^{9,25}$. The CLV3 dodecapeptide is a ligand for the leucine-rich receptor kinase CLV1 and related receptors, and functions in a negative feedback circuit with WUSCHEL (WUS), a homeobox transcription factor that promotes stem cell production in shoot meristems ${ }^{10,11}$. Mutations in CLV3 and its orthologs in many species cause meristem enlargement, which leads to tissue and organ overproliferation, or fasciation, phenotypes, especially in flowers ${ }^{9,10}$. We previously showed that $\operatorname{clv} 3$ mutations in the divergent species arabidopsis, Zea mays (maize), and Solanum lycopersicum (tomato) are buffered through redundancy, but through different mechanisms ${ }^{12}$. In arabidopsis, multiple $C L E$ family members partially suppress clv3 without changing their expression" ${ }^{12}$. In contrast to this "passive compensation", a similar partial suppression of clv3 mutations in maize (zmcle7) and tomato $(\operatorname{slclv} 3)$ is achieved by active compensation from closely related $C L V 3$ paralogs ${ }^{12}$. Though the mechanism of compensation is shared between maize and tomato, the paralogs involved arose through lineage-specific duplications, indicating independent evolution of active compensation. Thus, it remains unclear how states of active compensation are achieved in any lineage and whether they remain stable or continue to evolve as species diversify.

With several genetically tractable species, closely related Solanaceae family members comprise a useful system to track the evolution of the compensation relationship between CLV3 and its paralog. The compensating paralog in tomato, SlCLE9, originated from a duplication event just prior to diversification of the Solanales ${ }^{12}$. CRISPR-Cas 9 engineered slcle 9 mutations result in normal plants, but strongly enhance slclv3 due to loss of active compensation (Fig. 1a-c). Interestingly, our synteny analysis of 29 Solanaceae genomes capturing $\sim 30$ million years of evolution revealed several species that partially or completely lost their SlCLE9 orthologs (Fig. 1d and Supplementary Table 1) ${ }^{12}$. For example, whereas Physalis grisea (groundcherry) and 
87 Petunia hybrida (petunia) have SICLE9 orthologs, Capsicum annuum (pepper) harbors only 88 fragments of an SlCLE9 ortholog, indicating pseudogenization (Fig. 1d and Supplementary 89 Table 1) ${ }^{12}$. Both S. tuberosum (potato) and S. melongena (eggplant) lack SlCLE9 orthologs entirely, and this presence-absence variation extends to the genus level; in Nicotiana (tobacco), the SlCLE9 orthologs in N. tabacum and N. benthamiana were retained or pseudogenized, respectively (Fig. 1d and Supplementary Table 1).

Since active compensation is typically mediated by the existence of a close paralog ${ }^{6,16}$, we predicted that species that lost their SICLE9 orthologs would lack active compensation. However, in such species, compensation could also have evolved from one or more CLE homologs, which could potentially compensate passively (i.e. without transcriptional upregulation), as found in the Brassicaceae species Arabidopsis thaliana ${ }^{12}$. We tested compensation in the allotetraploid $N$. benthamiana, where CRISPR-Cas9 genome editing is highly efficient, but brings an added layer of genetic complexity from having two sub-genome copies (orthologs) of all genes, including $N b C L V 3(N b C L V 3 a \text { and } N b C L V 3 b)^{26}$. To test for loss of compensation in this species, we designed a multiplex CRISPR-Cas9 construct with eight gRNAs designed to target $N b C L V 3 a$ and $N b C L V 3 b$ (four gRNAs each; Fig 1e). We obtained five first-generation transgenic $\left(\mathrm{T}_{0}\right)$ plants, and unsurprisingly, all were chimeric (Supplementary Fig. 1a-c). Three of these plants exhibited severe fasciation phenotypes like tomato slclv3 slcle 9 double mutants, including thick stems and extreme overproliferation of floral organs, whereas the other two plants were less fasciated (Supplementary Fig. 1c-d). Though all plants were chimeric for mutations in $N b C L V 3 a$ and $N b C L V 3 b$, sequencing showed the three strongest mutants carried only mutated alleles of both genes, suggesting a null-equivalent phenotype similar to tomato slclv3 slcle9 double mutants (Fig. $1 c$ and Supplementary Fig. 1a-c). To confirm that $N b C L V 3 a$ and $N b C L V 3 b$ function in a canonical $C L V$-WUS negative feedback circuit, we evaluated expression of both genes from shoot apices of individual $\mathrm{T}_{0}$ plants compared to wild-type (WT). Consistently, both genes were upregulated, similar to SlCLV3 in slclv3 mutants (Supplementary Fig. 1e) ${ }^{12}$. Though the severity of the floral fasciation in the strongest $\mathrm{T}_{0}$ plants precluded recovery of mutant seeds, these observations supported the absence of active compensation in $N$. benthamiana. Importantly, we

116 fortuitously provided progeny populations that carried null alleles of $n b c l v 3 b$ and segregated for a 
118

mutants and showed that meristems were more than twice as large in these plants compared to $n b c l v 3 b$ single mutants and WT controls (Fig. 1j, k). Together, these results show that active compensation in the regulation of meristem maintenance was lost in $N$. benthamiana and also supports that conservation of active compensation in the Solanaceae requires retention of SICLE9 orthologs.

We next asked if compensation varies in lineages that retained their SICLE9 orthologs, and where allelic variation between these lineages could affect paralog function. Orthologous CLE pre-propeptide sequences are highly variable between species, but their dodecapeptides are more conserved $^{22,23}$. Indeed, while SlCLV3 and SlCLE9 ortholog dodecapeptide sequences were nearly invariant in the Solanaceae, we found widespread variation in the coding and putative cisregulatory regions of both genes, as determined by conserved non-coding sequence (CNS) analyses (Supplementary Fig. 2 and Supplementary Table 1). To assess active compensation in other Solanaceae species carrying SICLE9 orthologs, we took advantage of established CRISPRCas9 genome editing in petunia (Fig. 2a). Strikingly, the phenotypes of independently derived phclv3 null mutants were both substantially weaker than tomato slclv3 mutants (Fig. 1b, 2b-d). Although the primary shoot meristem was larger than WT meristems, $80 \%$ of phclv3 flowers produced wild-type organ numbers (Fig. 2c-f). Given that multiple attempts to generate pgcle 9 mutants were unsuccessful, we micro-dissected phclv3 meristems for RNA-sequencing to profile differentially expressed genes due to mutation of PhCLV3. Notably, out of all petunia CLE family members only PhCLE9 was dramatically upregulated ( $>15$-fold) (Fig. 2g, h and Supplementary Table 2), consistent with SICLE9 upregulation in tomato slclv3 mutants and suggesting active compensation in petunia is mediated by PhCLE9 and is stronger than in tomato.

Conservation of CLE dodecapeptide sequences is critical for proper ligand folding and receptor binding ${ }^{27,28}$. A single amino acid at position 6 distinguishes the petunia PhCLE9 and tomato SICLE9 dodecapeptides, and a deeper analysis of conservation revealed that all species from tomato and its wild relatives through Jaltomata sinuosa have a serine at this position, whereas all other Solanaceae except for a subset of tobacco species have a glycine (Fig. 3a, Supplementary Fig. 2c and Supplementary Table 1) ${ }^{12,22}$. Beyond the Solanaceae, this glycine is invariant in angiosperm CLV3 orthologs, is highly conserved in other CLE peptides, and is essential in Arabidopsis CLV3 and CLE41 peptides for precise binding to their receptors (Supplementary Fig. 2 and Supplementary Table 1) ${ }^{12,22,27-30}$. These observations suggested that other Solanaceae 
species with the conserved glycine in their SICLE9 orthologs might have more effective ligands, and would also be more potent compensators than tomato SICLE9. We tested this using CRISPRCas9 genome editing in groundcherry (Supplementary Fig. 3). Notably, null mutation of groundcherry pgclv3 resulted in only weak phenotypes similar to petunia phclv3 mutants (Fig 3be and Supplementary Fig. 3a, b). We also engineered homozygous pgcle 9 null mutations, which were nearly identical to wild-type (Fig. 3b-e and Supplementary Fig. 3c), and consistent with these weak effects, the sizes of primary shoot meristems in both mutants were largely unchanged (Fig. 3f, g). Importantly, as in tomato and in petunia, the expressions of both PgCLV3 and PgCLE9 were upregulated in pgclv3 meristems (Fig. 3h, i and Supplementary Table 3), and pgclv3 pgcle 9 double null mutants were severely fasciated, similar to tomato slclv3 slcle 9 double mutants, confirming conservation of active compensation (Fig. 3j, k and Supplementary Fig. 3d, e). Thus, while active compensation is conserved between tomato, petunia, and groundcherry, compensation from SlCLE9 orthologs in petunia and groundcherry is stronger than in tomato.

Our dissections of active compensation in tomato, petunia, and groundcherry suggested that the conserved glycine of the dodecapeptide is necessary for potent compensation. In further support, two conserved residues (Aspartic acid and Phenylalanine) in S1CLV1, which is the primary receptor of SICLV3 and SICLE9 ligands ${ }^{12}$, are critical for interaction with the sixth glycine of CLE peptides (Supplementary Fig. 4) ${ }^{29,30}$. Solanaceae CLV1 orthologs are invariant in these ligand binding residues (Supplementary Fig. 4). To test if the groundcherry and petunia orthologs of CLV1 (PgCLV1 and PhCLV1) are also the primary receptors for PgCLE9 and PhCLE9 as in tomato, we made double mutants between the weakly fasciated groundcherry pgclv1 and pgclv3 and also the weakly fasciated petunia phclv1 and phclv3 null mutants (Supplementary Fig. 5) ${ }^{31}$. Consistently, the double null mutants in both species matched the severe fasciation of groundcherry pgclv3 pgcle 9 double mutants, and importantly, also the tomato slclv1 slclv3 and slclv3 slcle9 double mutants (Fig. 1c, 3j and Supplementary Fig. 5c-e). These results support that the glycine to serine change in the tomato S1CLE9 dodecapeptide could be reducing binding affinity to S1CLV1, thus explaining weaker compensation in this species.

To test the significance of the glycine, we asked if the genomic sequence of PgCLE9 completely suppressed by the genomic sequence of SlCLV3 $\left(g S l C L V 3^{\text {SlCLV3 }}\right), g P g C L E 9^{\text {PgCLE9 }}$ had no effect (Fig. 4a, b and Supplementary Fig. 6a, b). Poor heterologous expression between 
groundcherry and tomato could explain this result, so we transformed slclv3 mutants with a construct expressing the groundcherry dodecapeptide from the genomic sequence of tomato SlCLE9 ( $g S l C L E 9^{P g C L E 9}$ ) (Fig. 4a, b and Supplementary Fig. 6a, b). Surprisingly, this construct also failed to complement, leading us to ask if strong active compensation depended on the conserved glycine as well as higher expression of dodecapeptides having the glycine. In support of this, in contrast to tomato, the fold-change increases in expression of both groundcherry $P g C L E 9$ and petunia $P h C L E 9$ were higher relative to upregulation of $C L V 3$ in their respective clv3 mutants (Fig. 2h, 3i). As the promoter of tomato $S l C L V 3$ is more transcriptionally responsive than the promoter of SlCLE9 to slclv3 mutations (Fig. 3h), we used a construct expressing the groundcherry dodecapeptide from SlCLV3 genomic sequence ( $g S l C L V 3^{\text {PgCLE9 }}$, which strongly suppressed slclv3 mutants. Notably, this complementation was slightly weaker than with gSlCLV3 ${ }^{\text {SlCLV3 }}$, consistent with active compensation from PgCLE9 and PhCLE9 dodecapeptides in groundcherry and petunia still permitting weak phenotypes of their respective $\operatorname{clv} 3$ mutants (Fig. 4a, b and Supplementary Fig. 6a, b). A construct expressing the tomato S1CLE9 dodecapeptide from the same $S l C L V 3$ genomic sequence $\left(g S l C L V 3^{S l C L E 9}\right)$ failed to complement, indicating that higher expression alone is insufficient (Fig. 4a, b and Supplementary Fig. 6a, b). Consistently, a weaker expression of PgCLE9 dodecapeptide ( $\left.g S l C L E 9^{S I C L E 9 S 6 G}\right)$ or a stronger expression of SlCLE9 dodecapeptide ( $\left.g S l C L V 3^{\text {SlCLE9}}-2\right)$ could only suppress slclv3 slcle9 double mutants to slclv3 single mutant phenotypes (Supplementary Fig. 6c, d). Altogether, our results show that changes in both the dodecapeptide and its expression explain evolutionary variation in the strength of compensation between tomato and its relatives groundcherry and petunia (Fig. 4c).

Here, we uncovered a dynamic evolution of paralogs interacting in an active compensation relationship. A first step of paralog diversification that can promote their preservation is 'compensatory drift', through which optimal levels of dosage-sensitive genes are maintained by reducing the expression of one paralog and elevating the other ${ }^{32}$. CLV3 orthologs are dosagesensitive ${ }^{33-35}$, and the consistently higher expression levels of Solanaceae CLV3 orthologs relative to SlCLE9 orthologs indicate that compensatory drift and active compensation emerged soon after

207 duplication (Fig. 2g, 3h). However, despite this expression rebalancing, we found that CLV3 208 compensation degraded multiple times during the Solanaceae family radiation over the last $\sim 30$ million years (Fig. 5). At one extreme, N. benthamiana, and likely other species that lost their SlCLE9 orthologs, completely lost active compensation and thus buffering of meristem 
211 homeostasis. In tomato, both coding and cis-regulatory changes weakened SlCLE9, and we

212 pinpointed a critical amino acid change that facilitated partial degradation of compensation from

213 the more potent ancestral state found in groundcherry and petunia (Fig. 5). Thus, the differential

214 accumulation of genetic variation between SICLE9 orthologs in these four Solanaceae species

215 resulted in both qualitative and quantitative differences in compensation potencies. Our finding of 216 extensive coding and cis-regulatory variation between SICLE9 orthologs suggests a range of

217 potencies could exist in Solanaceae CLV3 compensation (Supplementary Fig. 2 and

218 Supplementary Table 1). For example, even among tobacco species, while $N$. benthamiana lost 219 compensation, $N$. obtusifolia likely has strong compensation due to retention of a glycine220 containing SlCLE9 ortholog, and surprisingly, the sub-genome copies of SlCLE9 orthologs in N. 221 attenuata, N. tabacum, and N. tomentosiformis each have a glycine and a serine (Supplementary 222 Fig. 2c and Supplementary Table 1).

223 Differences in transcriptional control may play a larger role. Widespread variation in cis-

224 regulatory regions among tomato species suggests even greater variation between species in the 225 Solanaceae family ${ }^{36}$. Such diversity, both within and between genera (Supplementary Fig. 2a), 226 could result in differences in upregulation of SlCLE9 orthologs and phenotypes when CLV3 227 activity is compromised. Such a wide range of compensation strengths could be a foundation for 228 species-specific phenotypes. Notably, a structural variant that partially disrupts the promoter of 229 SlCLV3 is a major tomato domestication fruit size QTL, and we found that its severity was 230 mitigated by active compensation from SICLE9, resulting in a more moderate effect that may have 231 facilitated selection ${ }^{12,37}$. The increase in fruit size from this variant may not have emerged if the 232 ancestral version of SlCLE9 was retained in tomato, and moreover, in groundcherry and other 233 Solanaceae orphan crops with potent SlCLE9 orthologs, engineering mutations in CLV3 alone 234 would likely not benefit fruit size ${ }^{31,38}$. Beyond the Solanaceae, variation in meristem shape and 235 form is associated with morphological variation within and between species ${ }^{39-41}$. Such differences 236 could in part be based on variation in compensation between meristem homeostasis genes, which 237 could also influence phenotypic outcomes from engineered variation in CLV network genes ${ }^{33,35,42}$. 239 phenotypic changes caused by natural and engineered variation in other species and gene families. 240 The era of pan-genomes ${ }^{43-46}$ continues to uncover remarkable diversity in paralogs, including 
242 retained paralogs. Our findings show that such variation, much of which could be cryptic ${ }^{47-49}$, can 243 impact phenotypes in unpredictable and subtle ways when members of a gene family are mutated 244 within or between species. Revealing and dissecting diverse paralogous relationships can advance 245 our understanding of how dynamically evolving duplicated genes shape phenotypic variation 246 across short time scales, and improve predictability in trait engineering of both old and new crops. 


\section{Figure legends}

\section{Fig. 1. Loss of the tobacco SICLE9 ortholog abolished compensation.}

a, Shoot and inflorescence of tomato wild-type (WT). White arrowheads, inflorescences. b, Shoot and inflorescence of tomato slclv3. White arrowheads, inflorescences; red arrowheads, branches.

c, Side and top-down view of tomato slclv3 slcle 9 shoot, inflorescence/floral meristem, and primary inflorescence. The red arrowhead indicates a fasciated shoot stem. d, Presence-absence variation of SICLE9 orthologs in the Solanaceae. Phylogenic relationships of the indicated species are shown. The blue checkmarks and the red Xs indicate presence and absence of the orthologs, respectively. e, Gene structures, and CRISPR-generated mutations of NbCLV3a and NbCLV3b. Orange rectangles indicate the CLE dodecapeptides regions. Targeted guide RNA (gRNA) and protospacer-adjacent motif (PAM) sequences are highlighted in red and bold underlined, respectively. Blue letters and dashes indicate insertions and deletions, respectively. Numbers in parentheses represent gap lengths. DNA sequences of gRNA target site 2 for both $N b C L V 3 a$ and $N b C L V 3 b$ are identical. f, Shoot, flower, and fruit pod of tobacco WT. White arrowheads, flowers. g, Side and top-down views of $n b \operatorname{clv} 3 a / b$ null mutants showing the shoot and primary flower. Red arrowheads indicate fasciated primary shoot (left panel) and shoot branches (right panel). h, Sepal number of primary flower from tobacco WT, WT sibling plants (WT sibs) and $n b c l v 3 a / b$ plants. i, Branch number of WT, WT sibs and $n b c l v 3 a / b$. j, Primary shoot apical meristems from WT and $n b c l v 3 a / b$. Red dotted lines mark width and height for meristem size quantification. $7 \mathrm{~L}, 7^{\text {th }}$ leaf primordium. k, Quantification of meristem width and height from WT, WT sibs and $n b c l v 3 a / b$. Box plots, $25^{\text {th }}-75^{\text {th }}$ percentile; center line, median; whiskers, full data range in $\mathbf{h}, \mathbf{i}$ and $\mathbf{k}$. Exact sample sizes (n) for replicate types are indicated in $\mathbf{h}, \mathbf{i}$ and $\mathbf{k}$. Letters indicate significance groups at $P<0.01$ (One-way ANOVA and Tukey test) in $\mathbf{h}, \mathbf{i}$ and $\mathbf{k}$. WT sibs are a mix of $n b c l v 3 b$ and $n b c l v 3 a /+n b c l v 3 b$ genotypes, which show wild-type phenotypes in $\mathbf{h}, \mathbf{i}$ and $\mathbf{k}$. See Source Data 4.

275 a, Gene structure and sequences of two phclv3 null alleles. Guide RNA and PAM sequences are highlighted in red and bold underlined, respectively. The orange rectangles in the gene structures

277 represent the regions for CLE dodecapeptides. Numbers in parentheses represent gap lengths. Blue 278 dashes indicate deletions. b, Shoot of petunia WT and phclv3 plants. White arrowheads, flowers. 
c, Representative flowers and fruit pods of petunia WT and phclv3 plants. Red arrowheads mark petals or carpels. Percentages indicate the proportions of flower and pod phenotypes. d, Quantification of petal and carpel numbers of WT and phclv3. e, Primary shoot apical meristems from petunia WT and phclv3. Red dotted lines mark width and height for meristem size quantification. 22L, 22 $2^{\text {th }}$ leaf primordium. f, Quantification of meristem width and height from petunia WT and phclv3. g, Normalized read counts of PhCLV3 and PhCLE9 from WT and phclv3 meristems. Expression unit, fragments per kilobase of transcript per million (FPKM). h, Expression fold-change of $P h C L V 3$ and PhCLE9 relative to the normalized counts of WT from phclv3. Box plots, $25^{\text {th }}-75^{\text {th }}$ percentile; center line, median; whiskers, full data range in $\mathbf{d}, \mathbf{f}, \mathbf{g}$ and h. $P$ values (two-tailed, two-sample $t$-test) in $\mathbf{d}, \mathbf{f}, \mathbf{g}$ and $\mathbf{h}$. Exact sample sizes (n) are shown as discrete numbers in $\mathbf{d}, \mathbf{f}, \mathbf{g}$ and $\mathbf{h}$. Each replicate (n) is from 50-60 meristems in $\mathbf{g}$ and $\mathbf{h}$.

Fig. 3. A highly conserved dodecapeptide amino acid is associated with potent compensation in groundcherry.

a, CLE protein structure and dodecapeptide sequences of SlCLE9 and SlCLV3 orthologs in the Solanaceae. The sixth residue of the dodecapeptides are highlighted by red or blue bold font. b, Shoot and inflorescences of groundcherry WT, pgclv3 and pgcle 9 plants. Red arrowheads mark two side shoots that develop after single-flowered inflorescences. c, Representative flowers and fruits from groundcherry WT, pgclv3, and pgcle9 plants. Scale bar, $1 \mathrm{~cm}$. d, Representative flowers and fruits from tomato WT, slclv3, and slcle 9 plants. White arrowheads mark petals or locules. Scale bar, $1 \mathrm{~cm}$. e, Quantification and comparison of petal and locule numbers from groundcherry WT, pgclv3, pgcle9 and tomato WT, slclv3, and slcle9 plants. f, Primary shoot apical meristems from groundcherry WT, pgclv3, pgcle 9 and tomato WT, slclv3, and slcle9 plants. 7L, 8L: $7^{\text {th }}$ and $8^{\text {th }}$ leaf primordia, respectively. Red dotted lines indicate width and height for meristem size measurements, Scale bar, $200 \mu \mathrm{m}$. g, Quantification of meristem width and height from groundcherry WT, pgclv3, pgcle9, tomato WT, slclv3, and slcle9 plants. h, Normalized RNA-seq read counts of $S l C L V 3$, SlCLE9, PgCLV3, and PgCLE9 from tomato WT, slclv3, groundcherry WT and pgclv3 meristems. Expression units are counts per million (CPM) for tomato and transcripts per million (TPM) for groundcherry i, Expression fold-change of SlCLV3, SlCLE9, $P g C L V 3$, and PgCLE9 relative to the normalized counts of WT expressions of these genes in the indicated genotypes. j, Side and top-down views of a pgclv3 pgcle 9 double mutant shoot, 
inflorescence/floral meristem, and primary flower. Red arrowheads indicate branches that emerged after the primary flower. k, Branch number of WT, pgclv3, pgcle9, and pgclv3 pgcle9 plants. Box plots, $25^{\text {th }}-75^{\text {th }}$ percentile; center line, median; whiskers, full data range in $\mathbf{e}, \mathbf{g}, \mathbf{h}, \mathbf{i}$ and $\mathbf{k}$. The letters indicate the significance groups at $P<0.01$ (One-way ANOVA and Tukey test) in $\mathbf{e}, \mathbf{g}$ and k. $P$ values (two-tailed, two-sample $t$-test) in $\mathbf{h}$ and $\mathbf{i}$. Exact sample sizes (n) are shown in $\mathbf{e}, \mathbf{g}, \mathbf{h}$, $\mathbf{i}$ and $\mathbf{k}$. Each replicate (n) is from 30-35 meristems in $\mathbf{h}$ and $\mathbf{i}$.

Fig. 4. Variation in Solanaceae compensation is due to changes in both the SICLE9 ortholog dodecapeptide and its expression.

a, Diagrams of constructs used for complementation tests. gPgCLE9 ${ }^{\text {PgCLE9 }}$ (PgCLE9 genomic DNA). $g$ SlCLE $9^{\text {PgCLE9 }}$ (SlCLE9 genomic DNA including the sequence for PgCLE9 dodecapeptide). gSlCLV3 $3^{\text {PgCLE9 }}$ (SlCLV3 genomic DNA including the sequence for PgCLE9 dodecapeptide). gSlCLV3 $3^{\text {SlCLE9 }}$ (SlCLV3 genomic DNA including the sequence for SlCLE9 dodecapeptide). Black and orange rectangles mark the coding sequences and the dodecapeptide sequences, respectively. The numbers with minus (-) and plus (+) signs indicate the positions of the upstream sequences and the downstream sequences from the adenines of start codons, respectively. b, Locule number quantification from WT and slclv3 mutants compared to $\mathrm{T}_{1}$ transgenic plants of gSlCLV3 ${ }^{\text {SlCLV3 }}$, $g P g C L E 9^{P g C L E 9}, g S l C L E 9^{P g C L E 9}, g S l C L V 3^{P g C L E 9}$, and $g S l C L V 3^{S l C L E 9}$. Box plots, $25^{\text {th }}-75^{\text {th }}$ percentile; center line, median; whiskers, full data range. The letters indicate the significance groups at $P<$ 0.01 (One-way ANOVA and Tukey test). Exact sample sizes (n) are shown as discrete numbers. Data are based on at least 10 independent transgenic lines for each construct. c, A proposed model for differences in active compensation between tomato and groundcherry. The more potent active compensation in groundcherry compared to tomato is due to both the glycine-containing PgCLE9 dodecapeptide and its higher expression.

Fig. 5. Summary and model of the dynamic evolution of SICLV3 and SICLE9 orthologs and their compensation relationships in the Solanaceae. thickness represent gene expressions and their relative levels, respectively. Numbers above the arrows indicate hypothetical relative proportions of SlCLV3 and SlCLE9 ortholog expression levels. ' $\mathrm{G}$ ' and 'S' within the rectangles denote the sixth amino acid of each CLE dodecapeptide. Dashed 
341 rectangles mark deletions of the coding region, resulting in pseudogenes (pepper and tobacco) and

342 complete gene loss (eggplant, potato) in each genome. The red gradient bar reflects the loss of 343 active compensation and its degree, depending on the indicated genetic variation. 


\section{Methods}

\section{Plant materials and growth conditions.}

347 Seeds of petunia ( $P$. hybrida 'W115', Mitchel diploid) were provided by Prof. Yulong Guo, 348 Southwest University (Chongqing, China). Seeds of tobacco ( $N$. benthamiana), groundcherry $(P$. 349 grisea) and tomato (S. lycopersicum, cultivar M82) were from Cold Spring Harbor Laboratory 350 (CSHL) seed stocks. All seeds were sown directly in soil and grown in growth chambers, 351 greenhouses or fields at CSHL, New York, USA (tomato, tobacco, groundcherry) and Institute of 352 Genetics and Developmental Biology, Chinese Academy of Sciences, Beijing, China (petunia). 353 Briefly, groundcherry and tomato seedlings were grown in the greenhouse or field at CSHL as 354 described previously ${ }^{50}$. Tobacco plants were grown under long-day conditions (16 h light, $21^{\circ} \mathrm{C} / 8$ $355 \mathrm{~h}$ dark, $20^{\circ} \mathrm{C} ; 40-55 \%$ relative humidity; $75 \mu \mathrm{mol} \mathrm{m} \mathrm{m}^{-2} \mathrm{~s}^{-1}$ ) in the greenhouse at CSHL. Petunia 356 plants were grown under long-day conditions (16h light, $25^{\circ} \mathrm{C} / 8 \mathrm{~h}$ dark, $21^{\circ} \mathrm{C} ; 50-60 \%$ relative 357 humidity; $75 \mu \mathrm{mol} \mathrm{m} \mathrm{m}^{-2} \mathrm{~s}^{-1}$ ) in growth chambers and greenhouses at Institute of Genetics and 358 Developmental Biology, Chinese Academy of Sciences. All plants were grown under overhead 359 watering (tobacco) or drip irrigation (groundcherry, petunia and tomato), and standard fertilizer 360 regimes.

\section{CRISPR-Cas9 genome editing and plant transformation.}

363 Targeted mutagenesis using the CRISPR-Cas9 system for tobacco, groundcherry, and petunia were 364 performed as described previously ${ }^{31,51-57}$. Briefly, the binary vectors were constructed through 365 Golden Gate cloning as described ${ }^{51,58}$, and introduced into tobacco, groundcherry, and petunia by 366 Agrobacterium tumefaciens-mediated transformation as described ${ }^{52,53,57,59}$. First-generation 367 transgenic plants were transplanted in soil and genotyped to validate CRISPR-generated mutations by PCR and Sanger sequencing, as previously described ${ }^{37}$. All primer and gRNA sequences are included in Supplementary Table 4.

\section{Plant phenotyping and meristem imaging.}

372 All phenotypic quantification data on inflorescences and fruits were performed as previously 373 described ${ }^{12,37}$. Briefly, the phenotypic characterization was performed with biallelic or chimeric $374 \mathrm{~T}_{0}$ plants (tobacco), and non-transgenic homozygous plants (groundcherry, petunia, and tomato) 375 from backcrossing or selfing. CRISPR-generated null mutants of groundcherry and tomato were 
sprayed with $400 \mathrm{mgl}^{-1}$ kanamycin, and petunia were sprayed with $100 \mathrm{mgl}^{-1}$ kanamycin and genotyped by PCR to verify the absence of the transgenes. We manually counted the floral organs (petal and carpel/locule) from multiple inflorescence and plants. All the exact sample numbers of individual transgenic plants and aggregated organ quantifications are marked in the figures and are collated in the Source data. Meristem imaging and size quantification were conducted as described previously ${ }^{37,60}$. Briefly, the images of hand-dissected meristems were captured on a Nikon SMZ1500 (tomato), Nikon SMZ25 (groundcherry and tomato). Dissection and stereomicroscope imaging of petunia meristems were carried out under Olympus microscope (SteREO Discovery, v.12).

\section{RNA extraction, complementary DNA synthesis and quantitative real-time PCR (qPCR)}

RNA extraction and qPCR for tobacco, groundcherry and petunia were conducted as previously described with minor modification ${ }^{12,50}$. Briefly, for total RNA of the tobacco apices, the dissected shoot apices were extracted with RNeasy Plant Mini Kit (QIAGEN) according to the manufacturer's instructions. $1 \mu \mathrm{g}$ total RNA was used for reverse transcriptase PCR with the SuperScript III First-Strand Synthesis System (Invitrogen). qPCR was conducted in the iQ SYBR Green Supermix (Bio-Rad Laboratories) reaction system on the CFX96 Real-Time PCR Detection System (Bio-Rad) with gene-specific primers (Supplementary Table 4) ${ }^{61}$. For total RNA of the groundcherry meristems, the hand-dissected shoot apical meristems were extracted by the ARCTURUS PicoPure RNA Extraction Kit (Applied Biosystems). 30-35 meristems were collected for one replicate for each genotype. Total RNA of the petunia meristems was also extracted by the ARCTURUS PicoPure RNA Extraction Kit (Applied Biosystems). 50-60 meristems were collected for one replicate for each genotype.

401 The transcriptome data from tomato meristems were obtained from our previous RNA-seq data 402 deposited in the Sequence Read Archive project (SRP161864) and BioProject (PRJNA491365) ${ }^{12}$. 403 RNA-seq and differentially expressed genes (DEGs) analyses of groundcherry and petunia 404 meristems were performed as previously described with slight modification ${ }^{12}$. Briefly, the libraries 405 for RNA-sequencing (RNA-seq) were prepared by the KAPA mRNA HyperPrep Kit (Roche). The 
75-base sequencing was conducted on the Illumina sequencing platform (NextSeq, Mid-Output). Reads for the wild-type (WT) groundcherry and pgclv3 mutant were trimmed by quality using Trimmomatic v.0.32 (parameters: ILLUMINACLIP:TruSeq3-PE-2.fa:2:40:15:1:FALSE LEADING:30 TRAILING:30 MINLEN:50) ${ }^{62}$ and aligned to the reference transcriptome assembly of groundcherry ${ }^{31}$ for quantification using 'kallisto quant' (v0.46.2, bootstrap: 100)63. Kallisto quantification results were used as inputs for 'sleuth' in R to get normalized estimated counts for each transcript ${ }^{64}$. For RNA-seq of petunia meristems, the libraries were prepared by SMARTer Ultra Low Input RNA for Sequencing Kit (Clontech). The quality of each library was validated with a 2100 Bioanalyzer (Agilent Technologies). Paired-end 150-base sequencing was conducted on the Illumina NovaSeq 6000 sequencing platform (NextSeq, Mid-Output). Reads for the WT

417 petunia and phclv3 mutant were trimmed by quality using Trimmomatic v0.36 (ILLUMINACLIP:adapter.fa:2:30:10 LEADING:20 TRAILING:20 SLIDINGWINDOW:4:15 MINLEN:36) ${ }^{62}$ and aligned to the reference genome sequence of petunia ${ }^{65}$ using hisat2 v2.1.0 with default parameters ${ }^{66}$. Alignments were sorted with samtools v1.8 $8^{67}$ and the RNA-seq reads were assembled using StringTie (v2.0.3) with default parameters ${ }^{68}$. To verify and annotate the transcript of petunia PhCLE9 (Peaxi162Scf00429:766800-783916), orthologous Blast was performed using tomato SICLE9 as a bait and the resulting transcript was confirmed by PCR amplification followed by Sanger sequencing (see Supplementary Dataset 2). The expected read counts and fragments per kilobase of transcript per million mapped reads (FPKM) were also calculated using SringTie $(\mathrm{v} 2.0 .3)^{68}$. The statistical analyses for groundcherry and petunia data were performed in R(v3.5.2) (RStudio (v.1.1.463)) and R(v4.0.3), respectively ${ }^{69,70}$. Significant differential expression between groundcherry WT and pgclv3 mutant was identified with sleuth ${ }^{64}$ using $q$-value $\leq 0.01$ cut-offs. Significant differential expression between petunia WT and phclv3 mutant was confirmed with DESeq $2^{64,71}$ using $q$-value $\leq 0.05$ and $\mid \log 2$ ratio $\mid \geq 1$.

\section{Transgenic complementation of PgCLE9, SICLV3 and SICLE9.}

433 The transgenic lines and genomic DNA sequence for $g S l C L V 3^{\text {SlCLV3 }}$ and $g S l C L V 3^{\text {SlCLE9 }}$ were 434 procured from our previous study ${ }^{12}$. The genomic DNA sequences of PgCLE9 consisted of $435 \mathrm{gPgCLE} 9^{P g C L E 9} 4471$ base pair (bp) in total with 3394 bp upstream, 548 bp of coding sequence containing introns, and 529 bp downstream. The genomic DNA sequences of SlCLE9 consisted of gSlCLE9 SICLE9 $^{4} 140 \mathrm{bp}$ in total with $3263 \mathrm{bp}$ upstream, 403 bp of coding sequence containing 
introns, and $474 \mathrm{bp}$ downstream. Site-directed mutageneses were performed to substitute the S1CLE9 dodecapeptide into PgCLE9 within gSlCLE9SICLE9 $\left(\right.$ gSlCLE9 ${ }^{\text {PgCLE9 }}$ ) and the SICLV3 dodecapeptide into PgCLE9 within $g S l C L V 3^{S l C L V 3}$ ( $g S l C L V 3^{\text {PgCLE9 }}$ ). The PCR products were amplified from the vectors including the genomic region of SlCLV3 (pICH47742-gSlCLV3 $3^{\text {SICLV3 }}$ ) and SlCLE9 (pICH47742- SSlCLE9SICLE9) with overlapping primers (Supplementary Table 4) using KOD One ${ }^{\mathrm{TM}}$ PCR Master Mix (TOYOBO). Then, the amplified PCR products were digested using DpnI (New England Biolabs) and transformed into DH5a competent cells. The sequences of the resulting plasmids were confirmed by Sanger sequencing with multiple primers (Supplementary Table 4). The Level 1 vectors (pICH47742-gPgCLE9 ${ }^{\text {PgCLE9 }}$, gSlCLE9 $9^{\text {PCLE9 }}$ and gSlCLV3 $3^{P g C L E 9}$ ) were assembled with the construct pICH47732-NOSpro::NPTII into the binary vector pICSL4723 through Golden Gate cloning as previously described ${ }^{51,58,72}$. The binary vectors were introduced into the tomato slclv3 mutant by Agrobacterium tumefaciens-mediated transformation as previously described ${ }^{53}$. The genomic DNA sequences of SlCLV3 consisted of

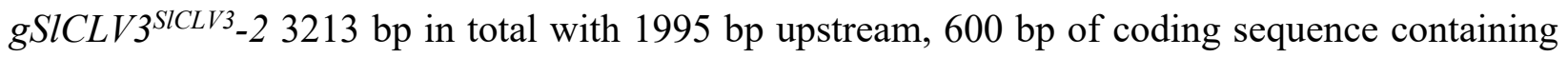
introns, and $618 \mathrm{bp}$ downstream. The genomic DNA sequences of SlCLE9 consisted of gSlCLE9SICLE9-2 2740 bp in total with 1996 bp upstream, 403 bp of coding sequence containing introns, and $341 \mathrm{bp}$ downstream. Site-directed mutagenesis was performed to substitute the SICLV3 dodecapeptide into S1CLE9 within gSlCLV3 ${ }^{\text {SlCLV3 }}\left(\right.$ gSlCLV3 $\left.{ }^{\text {SlCLE9}}-2\right)$ and the S1CLE9 dodecapeptide into S1CLE9 ${ }^{\text {S6G }}$ within SSICLE9 $^{\text {SICLE9 }}$ ( $\left(\right.$ SICLE9 ${ }^{S I C L E 9 S 6 G}$ ). The PCR products were amplified from the vectors including the genomic region of SlCLV3 (pDONOR221-gSlCLV3 ${ }^{\text {SlCLV3 }}$ 2) and SlCLE9 (pDONOR221- gSlCLE9SICLE9-2) with overlapping primers (Supplementary Table 4) using KOD One ${ }^{\mathrm{TM}}$ PCR Master Mix (TOYOBO). Then, the amplified PCR products were digested using DpnI (New England Biolabs) and transformed into DH5a competent cells. The sequences of the resulting plasmids were confirmed by Sanger sequencing with multiple primers (Supplementary Table 4), and colonies were recombined into binary vector pGWB40173 for transgenic complementation. The binary vectors were introduced into the tomato slclv 3 slcle 9 464 double mutant by Agrobacterium tumefaciens-mediated transformation as previously described ${ }^{53}$. 465 Transgenic lines were confirmed by PCR and kanamycin resistance, and at least three independent transgenic lines from each construct were used for data collection (see Source Data). 
469 Analysis of conserved non-coding sequences (CNSs) is a common approach to identify putative 470 cis-regulatory sequences of genes (e.g. promoters, enhancers). Solanaceae orthologous genes of 471 SlCLV3 and SlCLE9 for synteny analysis and CNSs in the promoter regions surrounding the 472 orthologs of SlCLV3 and SlCLE9 were identified using our previously developed Conservatory 473 algorithm, using default parameters ${ }^{74}$. In parallel, all of the genomes were scanned with tBLASTn 474 to find mis- or unannotated protein coding regions for each gene. CNSs in the promoter regions 475 were called by Conservatory using default parameters ${ }^{74}$. To calculate protein identity percentages 476 and dodecapeptide identity percentages, protein sequences were aligned by MAFFT (v.7.45) using 477 BLOSUM62 matrix and 'E-INS-i' and 'G-INS-i' algorithm respectively ${ }^{75}$.

\section{Statistical analyses.}

480 Statistical calculations were conducted using R(v3.5.2 and v4.0.3) ${ }^{69}$ and Microsoft Excel, as 481 previously described ${ }^{50}$. Statistical analyses were performed using a two-tailed, two-sample $t$-test 482 and a one-way analysis of variance (ANOVA) with Tukey test. The exact sample sizes (n) and all 483 raw data for each experimental group/condition are given as discrete numbers in each figure panel 484 and Source data. Additional information is available in the Nature Research Reporting Summary, 485 which includes statements on statistics, software used and data availability.

\section{Data availability}

488 Raw data and information for CRISPR-generated alleles, all quantifications, synteny analysis, and 489 exact $P$ values (One-way ANOVA and Tukey test) are in Source data. The raw Sanger sequence 490 traces for edited sequences are in Supplementary Dataset 1. The groundcherry and petunia 491 BioProject accession numbers are PRJNA704671 and PRJNA750419, respectively. 


\section{References}

495 1. Conrad, B. \& Antonarakis, S. E. Gene Duplication: A Drive for Phenotypic Diversity and 496 Cause of Human Disease. Annu. Rev. Genomics Hum. Genet. 8, 17-35 (2007).

497 2. Carretero-Paulet, L. \& Fares, M. A. Evolutionary Dynamics and Functional Specialization of 498 Plant Paralogs Formed by Whole and Small-Scale Genome Duplications. Mol. Biol. Evol. 29, $499 \quad 3541-3551(2012)$.

500 3. Panchy, N., Lehti-Shiu, M. \& Shiu, S.-H. Evolution of Gene Duplication in Plants1[OPEN]. $501 \quad$ Plant Physiol. 171, 2294-2316 (2016).

4. Copley, S. D. Evolution of new enzymes by gene duplication and divergence. FEBS J. 287, $1262-1283(2020)$.

5. Kuzmin, E., Taylor, J. S. \& Boone, C. Retention of duplicated genes in evolution. Trends

505 Genet., (2021). doi: 10.1016/j.tig.2021.06.016.

6. Diss, G., Ascencio, D., DeLuna, A. \& Landry, C. R. Molecular mechanisms of paralogous compensation and the robustness of cellular networks. J. Exp. Zoolog. B Mol. Dev. Evol. 322, 488-499 (2014).

7. Kafri, R., Springer, M. \& Pilpel, Y. Genetic Redundancy: New Tricks for Old Genes. Cell

510 136, 389-392 (2009).

511 8. DeLuna, A., Springer, M., Kirschner, M. W. \& Kishony, R. Need-Based Up-Regulation of 512 Protein Levels in Response to Deletion of Their Duplicate Genes. PLOS Biol. 8, e1000347 $513 \quad$ (2010).

514 9. Somssich, M., Je, B. I., Simon, R. \& Jackson, D. CLAVATA-WUSCHEL signaling in the 515 shoot meristem. Development 143, 3238-3248 (2016).

516 10. Fletcher, J. C. The CLV-WUS Stem Cell Signaling Pathway: A Roadmap to Crop Yield 517 Optimization. Plants 7, 87 (2018). 
518 11. Soyars, C. L., James, S. R. \& Nimchuk, Z. L. Ready, aim, shoot: stem cell regulation of 519 the shoot apical meristem. Curr. Opin. Plant Biol. 29, 163-168 (2016).

520 12. Rodriguez-Leal, D. et al. Evolution of buffering in a genetic circuit controlling plant stem 521 cell proliferation. Nat. Genet. 51, 786-792 (2019).

522 13. Jiao, W.-B. et al. The Evolutionary Dynamics of Genetic Incompatibilities Introduced by 523 Duplicated Genes in Arabidopsis thaliana. Mol. Biol. Evol. 38, 1225-1240 (2021).

524 14. Innan, H. \& Kondrashov, F. The evolution of gene duplications: classifying and 525 distinguishing between models. Nat. Rev. Genet. 11, 97-108 (2010).

526 15. Albalat, R. \& Cañestro, C. Evolution by gene loss. Nat. Rev. Genet. 17, 379-391 (2016).

527 16. El-Brolosy, M. A. \& Stainier, D. Y. R. Genetic compensation: A phenomenon in search 528 of mechanisms. PLOS Genet. 13, e1006780 (2017).

529 17. Hanada, K. et al. Functional Compensation of Primary and Secondary Metabolites by 530 Duplicate Genes in Arabidopsis thaliana. Mol. Biol. Evol. 28, 377-382 (2011).

531 18. Kafri, R., Bar-Even, A. \& Pilpel, Y. Transcription control reprogramming in genetic 532 backup circuits. Nat. Genet. 37, 295-299 (2005).

533 19. DeLuna, A. et al. Exposing the fitness contribution of duplicated genes. Nat. Genet. 40, $534 \quad 676-681(2008)$

535 20. Freeling, M. Bias in Plant Gene Content Following Different Sorts of Duplication: 536 Tandem, Whole-Genome, Segmental, or by Transposition. Annu. Rev. Plant Biol. 60, 433$537453(2009)$.

538 21. Vavouri, T., Semple, J. I. \& Lehner, B. Widespread conservation of genetic redundancy 539 during a billion years of eukaryotic evolution. Trends Genet. 24, 485-488 (2008). 
540 22. Goad, D. M., Zhu, C. \& Kellogg, E. A. Comprehensive identification and clustering of

541 CLV3/ESR-related (CLE) genes in plants finds groups with potentially shared function. New

$542 \quad$ Phytol. 216, 605-616 (2017).

543 23. Fletcher, J. C. Recent Advances in Arabidopsis CLE Peptide Signaling. Trends Plant Sci.

$544 \quad 25,1005-1016(2020)$.

545 24. Hirakawa, Y. \& Sawa, S. Diverse function of plant peptide hormones in local signaling 546 and development. Curr. Opin. Plant Biol. 51, 81-87 (2019).

547 25. Whitewoods, C. D. et al. CLAVATA Was a Genetic Novelty for the Morphological

548 Innovation of 3D Growth in Land Plants. Curr. Biol. 28, 2365-2376.e5 (2018).

549 26. Stuttmann, J. et al. Highly efficient multiplex editing: one-shot generation of $8 \times$

550 Nicotiana benthamiana and 12× Arabidopsis mutants. Plant J. 106, 8-22 (2021).

551 27. Fiers, M. et al. The CLAVATA3/ESR Motif of CLAVATA3 Is Functionally Independent 552 from the Nonconserved Flanking Sequences. Plant Physiol. 141, 1284-1292 (2006).

553 28. Ogawa, M., Shinohara, H., Sakagami, Y. \& Matsubayashi, Y. Arabidopsis CLV3 Peptide 554 Directly Binds CLV1 Ectodomain. Science 319, 294-294 (2008).

555 29. Zhang, H., Lin, X., Han, Z., Qu, L.-J. \& Chai, J. Crystal structure of PXY-TDIF complex 556 reveals a conserved recognition mechanism among CLE peptide-receptor pairs. Cell Res. 26, $557 \quad 543-555(2016)$.

558 30. Li, Z., Chakraborty, S. \& Xu, G. Differential CLE peptide perception by plant receptors 559 implicated from structural and functional analyses of TDIF-TDR interactions. PLOS ONE 12, $560 \quad$ e0175317 (2017).

561 31. Lemmon, Z. H. et al. Rapid improvement of domestication traits in an orphan crop by 562 genome editing. Nat. Plants 4, 766-770 (2018). 
563 32. Thompson, A., Zakon, H. H. \& Kirkpatrick, M. Compensatory Drift and the Evolutionary

564 Dynamics of Dosage-Sensitive Duplicate Genes. Genetics 202, 765-774 (2016).

565 33. Rodríguez-Leal, D., Lemmon, Z. H., Man, J., Bartlett, M. E. \& Lippman, Z. B.

566 Engineering Quantitative Trait Variation for Crop Improvement by Genome Editing. Cell 171, $567 \quad$ 470-480.e8 (2017).

568 34. Wang, X. et al. Dissecting cis-regulatory control of quantitative trait variation in a plant 569 stem cell circuit. Nat. Plants 7, 419-427 (2021).

570 35. Liu, L. et al. Enhancing grain-yield-related traits by CRISPR-Cas9 promoter editing of 571 maize CLE genes. Nat. Plants 7, 287-294 (2021).

572 36. Alonge, M. et al. Major Impacts of Widespread Structural Variation on Gene Expression 573 and Crop Improvement in Tomato. Cell 182, 145-161.e23 (2020).

574 37. Xu, C. et al. A cascade of arabinosyltransferases controls shoot meristem size in tomato. $575 \quad$ Nat. Genet. 47, 784-792 (2015).

576 38. Samuels, J. Biodiversity of Food Species of the Solanaceae Family: A Preliminary 577 Taxonomic Inventory of Subfamily Solanoideae. Resources 4, 277-322 (2015).

578 39. Leiboff, S. et al. Genetic control of morphometric diversity in the maize shoot apical 579 meristem. Nat. Commun. 6, 8974 (2015).

580 40. Leiboff, S., DeAllie, C. K. \& Scanlon, M. J. Modeling the Morphometric Evolution of 581 the Maize Shoot Apical Meristem. Front. Plant Sci. 7, 1651 (2016).

582 41. Schnablová, R., Herben, T. \& Klimešová, J. Shoot apical meristem and plant body 583 organization: a cross-species comparative study. Ann. Bot. 120, 833-843 (2017).

584 42. Yang, Y. et al. Precise editing of CLAVATA genes in Brassica napus L. regulates 585 multilocular silique development. Plant Biotechnol. J. 16, 1322-1335 (2018). 
43. Khan, A. W. et al. Super-Pangenome by Integrating the Wild Side of a Species for

44. Della Coletta, R., Qiu, Y., Ou, S., Hufford, M. B. \& Hirsch, C. N. How the pan-genome 589 is changing crop genomics and improvement. Genome Biol. 22, 3 (2021).

45. Bayer, P. E., Golicz, A. A., Scheben, A., Batley, J. \& Edwards, D. Plant pan-genomes are 591 the new reference. Nat. Plants 6, 914-920 (2020).

592 46. Sherman, R. M. \& Salzberg, S. L. Pan-genomics in the human genome era. Nat. Rev. 593 Genet. 21, 243-254 (2020).

594 47. Paaby, A. B. \& Rockman, M. V. Cryptic genetic variation: evolution's hidden substrate. $595 \quad$ Nat. Rev. Genet. 15, 247-258 (2014).

596 48. Wagner, A. The molecular origins of evolutionary innovations. Trends Genet. 27, 397$597 \quad 410(2011)$.

598 49. Zheng, J., Payne, J. L. \& Wagner, A. Cryptic genetic variation accelerates evolution by 599 opening access to diverse adaptive peaks. Science 365, 347-353 (2019).

600 50. Kwon, C.-T. et al. Rapid customization of Solanaceae fruit crops for urban agriculture. $601 \quad$ Nat. Biotechnol. 38, 182-188 (2020).

602 51. Brooks, C., Nekrasov, V., Lippman, Z. B. \& Van Eck, J. Efficient gene editing in tomato 603 in the first generation using the clustered regularly interspaced short palindromic 604 repeats/CRISPR-associated9 system. Plant Physiol. 166, 1292-1297 (2014).

605 52. Swartwood, K. \& Van Eck, J. Development of plant regeneration and Agrobacterium 606 tumefaciens-mediated transformation methodology for Physalis pruinosa. Plant Cell Tissue 607 Organ Cult. PCTOC 137, 465-472 (2019). 
608 53. Van Eck, J., Keen, P. \& Tjahjadi, M. Agrobacterium tumefaciens-Mediated

609 Transformation of Tomato. in Transgenic Plants: Methods and Protocols (eds. Kumar, S.,

610 Barone, P. \& Smith, M.) 225-234 (Springer New York, 2019). doi:10.1007/978-1-4939-8778-

$6118+16$.

612 54. Zhang, B., Yang, X., Yang, C., Li, M. \& Guo, Y. Exploiting the CRISPR/Cas9 System

613 for Targeted Genome Mutagenesis in Petunia. Sci. Rep. 6, 20315 (2016).

614 55. Zhang, B. et al. CRISPR/Cas9-mediated targeted mutation reveals a role for AN4 rather

615 than DPL in regulating venation formation in the corolla tube of Petunia hybrida. Hortic. Res.

$616 \quad 8,1-9(2021)$.

617 56. Jiang, W. et al. Demonstration of CRISPR/Cas9/sgRNA-mediated targeted gene

618 modification in Arabidopsis, tobacco, sorghum and rice. Nucleic Acids Res. 41, e188-e188

619 (2013).

620 57. Gantner, J., Ordon, J., Kretschmer, C., Guerois, R. \& Stuttmann, J. An EDS1-SAG101

621 Complex Is Essential for TNL-Mediated Immunity in Nicotiana benthamiana. Plant Cell 31,

$622 \quad 2456-2474(2019)$.

623 58. Werner, S., Engler, C., Weber, E., Gruetzner, R. \& Marillonnet, S. Fast track assembly of 624 multigene constructs using Golden Gate cloning and the MoClo system. Bioeng. Bugs 3, 38$62543(2012)$

626 59. van der Meer, I. M. Agrobacterium-Mediated Transformation of Petunia Leaf Discs. in

627 Plant Cell Culture Protocols (eds. Loyola-Vargas, V. M. \& Vázquez-Flota, F.) 265-272

628 (Humana Press, 2006). doi:10.1385/1-59259-959-1:265. 
629 60. Park, S. J., Jiang, K., Schatz, M. C. \& Lippman, Z. B. Rate of meristem maturation

630 determines inflorescence architecture in tomato. Proc. Natl. Acad. Sci. U. S. A. 109, 639-644

631 (2012).

632 61. Liu, D. et al. Validation of Reference Genes for Gene Expression Studies in Virus-

633 Infected Nicotiana benthamiana Using Quantitative Real-Time PCR. PLOS ONE 7, e46451

634 (2012).

635 62. Bolger, A. M., Lohse, M. \& Usadel, B. Trimmomatic: a flexible trimmer for Illumina

636 sequence data. Bioinformatics 30, 2114-2120 (2014).

637 63. Bray, N. L., Pimentel, H., Melsted, P. \& Pachter, L. Near-optimal probabilistic RNA-seq 638 quantification. Nat. Biotechnol. 34, 525-527 (2016).

639 64. Pimentel, H., Bray, N. L., Puente, S., Melsted, P. \& Pachter, L. Differential analysis of 640 RNA-seq incorporating quantification uncertainty. Nat. Methods 14, 687-690 (2017).

641 65. Bombarely, A. et al. Insight into the evolution of the Solanaceae from the parental 642 genomes of Petunia hybrida. Nat. Plants 2, 1-9 (2016).

643 66. Kim, D., Langmead, B. \& Salzberg, S. L. HISAT: a fast spliced aligner with low memory 644 requirements. Nat. Methods 12, 357-360 (2015).

645 67. Li, H. et al. The Sequence Alignment/Map format and SAMtools. Bioinformatics 25, $646 \quad 2078-2079(2009)$.

647 68. Pertea, M. et al. StringTie enables improved reconstruction of a transcriptome from 648 RNA-seq reads. Nat. Biotechnol. 33, 290-295 (2015).

649 69. R Core Team (2015). R: A language and environment for statistical computing. (R 650 Found. Stat. Comput. Vienna, Austria.). 
651 70. LoVerso, P. R. \& Cui, F. A Computational Pipeline for Cross-Species Analysis of RNA-

652 seq Data Using R and Bioconductor. Bioinforma. Biol. Insights 9, 165-174 (2015).

653 71. Love, M. I., Huber, W. \& Anders, S. Moderated estimation of fold change and dispersion 654 for RNA-seq data with DESeq2. Genome Biol. 15, 550 (2014).

655 72. Weber, E., Engler, C., Gruetzner, R., Werner, S. \& Marillonnet, S. A Modular Cloning 656 System for Standardized Assembly of Multigene Constructs. PLoS ONE 6, e16765 (2011).

657 73. Nakagawa, T. et al. Improved Gateway binary vectors: high-performance vectors for 658 creation of fusion constructs in transgenic analysis of plants. Biosci. Biotechnol. Biochem. 71, $659 \quad 2095-2100(2007)$.

660 74. Hendelman, A. et al. Conserved pleiotropy of an ancient plant homeobox gene uncovered 661 by cis-regulatory dissection. Cell 184, 1724-1739.e16 (2021).

662 75. Katoh, K. \& Standley, D. M. MAFFT multiple sequence alignment software version 7: 663 improvements in performance and usability. Mol. Biol. Evol. 30, 772-780 (2013). 


\section{Acknowledgements}

666 We thank members of the Lippman laboratory for comments and discussions, and also critical

667 friends Y. Eshed and M. Bartlett. We thank S. Soyk for discussions and initial peptide sequence 668 analysis in tomato, and J. Kim and A. Krainer for technical support. We thank A. Horowitz Doyle, 669 K. Swartwood, M. Tjahjadi, L. Randall, and P. Keen from the Van Eck lab for performing tobacco, 670 groundcherry and tomato transformations. We thank T. Mulligan, K. Schlecht, A. Krainer and S. 671 Qiao for assistance with plant care. This research was supported by National Natural Science 672 Foundation of China (grant no. 31972423 and 31991183) and Chinese Academy of Sciences (grant 673 no. 153E11KYSB20180019) to C.X., the Howard Hughes Medical Institute, an Agriculture and 674 Food Research Initiative competitive grant from the USDA National Institute of Food and 675 Agriculture (grant no. 2016-67013-24452) and the National Science Foundation Plant Genome 676 Research Program (grant no. IOS-1732253 and IOS-1546837) to Z.B.L.

\section{Author contributions}

679 C.-T.K. designed the research and conducted the experiments, prepared the figures and wrote the 680 manuscript. L.T. performed the petunia CRISPR experiments, tomato transgenic complementation 681 tests, genetic, RNA-seq and phenotypic analyses. X.W. performed the groundcherry RNA-seq, 682 phenotypic analyses, and wrote the manuscript. I.G performed the tobacco genetic and phenotypic 683 analyses. A.H. performed CLE family analyses. G.R. characterized CRISPR mutations. J.V.E 684 generated transgenic plants and CRISPR lines. C.X. supervised and led the petunia CRISPR 685 experiments and tomato transgenic complementation tests, genetic, RNA-seq and phenotypic 686 analyses, contributed ideas and edited the manuscript. Z.B.L. conceived and led the research, 687 supervised and performed the experiments, prepared the figures and wrote the manuscript. All 688 authors read, edited, and approved the manuscript.

\section{Competing interests}

691 The authors declare that they have no competing interests. 


\section{Additional information}

694 Supplementary figure legends

Supplementary Fig. 1. CRISPR-generated mutations of the tobacco $N b C L V 3 a$ and $N b C L V 3 b$ genes.

a, CRISPR-generated sequences of $n b c l v 3 a$ mutant alleles. $\mathbf{b}$, CRISPR-generated sequences of $n b c l v 3 b$ mutant alleles. Guide RNA and PAM sequences are highlighted in red and bold underlined, respectively. Blue letters and dashes indicate insertions and deletions, respectively. Numbers in parentheses represent gap lengths. c, Shoots and inflorescences of $n b c l v 3 a / b \mathrm{~T}_{0}$ plants. Three strong lines $\left(n b c l v 3 a / b^{C R-3-T 0}, n b c l v 3 a / b^{C R-4-T 0}\right.$ and $\left.n b c l v 3 a / b^{C R-5-T 0}\right)$ show similar phenotypes compared to null $n b c l v 3 a / b$ mutants in Fig. 1g. Weak $\left(n b c l v 3 a / b^{C R-6-T 0}\right)$ and moderate $\left(n b c l v 3 a / b^{C R-7-T O}\right)$ lines show regular shoot architecture but fasciated floral organs. White arrowheads indicate flowers. d, Sepal number of weak and moderate $n b c l v 3 a / b \mathrm{~T}_{0}$ plants. e, Relative expressions of $N b C L V 3 a$ and $N b C L V 3 b$ in shoot apices from $n b c l v 3 a / b \mathrm{~T}_{0}$ chimeric mutants, normalized to $N b P P 2 A$. Dashed line, value ' 1 ' on the y-axis. Three and one biological replicates for WT and $n b c l v 3 a / b$ plants, respectively; two or three technical replicates included. Box plots, $25^{\text {th }}-75^{\text {th }}$ percentile; center line, median; whiskers, full data range in $\mathbf{d}$ and $\mathbf{e}$. The letters indicate the significance groups at $P<0.01$ (One-way ANOVA and Tukey test) in $\mathbf{d}$ and e. The exact sample sizes (n) are shown as discrete numbers in $\mathbf{d}$ and $\mathbf{e}$.

Supplementary Fig. 2. Conserved noncoding sequence (CNS) analysis of the promoter

\section{regions of SICLV3 and SICLE9 orthologs in the Solanaceae family.}

a, Conservatory analysis of Solanaceae $C L V 3$ and $C L E 9$ promoters. Purple boxes define highly similar regions of each gene's orthologs in the indicated species, and dark purple boxes define

717 highly similar regions of the paralogous gene (e.g. CLV3B or CLE9B) in the indicated species.

718 Green boxes define Solanaceae CNSs. b, Multiple alignment and logo sequences of SICLV3

719 dodecapeptide orthologs in the Solanaceae family. c, Multiple alignment and logo sequences of 720 SlCLE9 dodecapeptide orthologs in the Solanaceae family. 
Supplementary Fig. 3. CRISPR-generated mutations of groundcherry PgCLV3 and PgCLE9.

a, Gene structure and sequences of pgclv3 CRISPR mutants. b, Flowers and fruits of pgclv3. White arrowheads mark petals or locules. Percentages indicate the proportions of flower and fruit phenotypes. Scale bar, $1 \mathrm{~cm}$. c, Gene structure and sequences of pgcle9 CRISPR mutants. The orange rectangles in the gene structures indicate the regions of the CLE dodecapeptides in a and c. Guide RNA and PAM sequences are highlighted in red and bold underlined, respectively, in a and c. Blue letters and dashes indicate insertions and deletions, respectively, in a and c. Numbers in parentheses represent gap lengths in a and c. d, Shoot and an extremely fasciated primary flower of the pgclv3 pgcle9 double mutant. e, Development of extra shoots (S) from the primary shoot and apex of a pgclv3 pgcle9 double mutant. L, leaf petioles.

Supplementary Fig. 4. Sequence alignments of CLV1 receptor homologs.

a, Alignment of the Solanaceae CLV1 protein sequences. Red letters indicate the two ultraconserved amino acids involved in the physical binding of CLE dodecapeptides. $\mathbf{b}$, Alignment of CLV1 homologs in angiosperms. All the sequences are from the Phytozome v12.1 database (phytozome.jgi.doe.gov). Yellow highlights mark the conserved Asp and Phe. Detailed sequence information is shown in Supplementary Dataset 3. mutants are severely fasciated like tomato slclv1 slclv3 double mutants.

a, Gene structure and sequences of two phclv1 CRISPR mutants. Guide RNA and PAM sequences are highlighted in red and bold underlined, respectively. Blue letters and dashes indicate insertions and deletions, respectively. Numbers in parentheses represent gap lengths. b, Flowers, fruits/pods, and meristems from pgclv1, phclv1, and slclv1 single mutants. White arrowheads mark petals or locules. $7 \mathrm{~L}, 7^{\text {th }}$ leaf primordium, $8 \mathrm{~L}, 8^{\text {th }}$ leaf primordium. $22 \mathrm{~L}, 22^{\text {th }}$ leaf primordium. C, Side and top-down views of a pgclv1 pgclv3 double mutant shoot, inflorescence/floral meristem, and primary inflorescence. $6 \mathrm{~L}, 6^{\text {th }}$ leaf primordium. D, Side and top-down views of a phclv1 phclv3 double mutant shoot and primary flower. E, Side and top-down views of a slclv1 slclv3 double mutant shoot, flower, vegetative meristem and primary inflorescence. Fasciated flowers and vegetative meristems are shown in insets of $\mathbf{c}$ and $\mathbf{e}$. f, g, Petal (f) and locule (g) numbers of groundcherry WT, pgclv1, pgclv1 pgclv3, pgclv1 pgcle9, and petunia WT, phclv1, and tomato WT, 
753 slclv1, slclv1 slclv3, and slclv1 slcle9. Not that all three Solanaceae clv1 single mutant fasciation

754 phenotypes are similarly weak. Box plots, $25^{\text {th }}-75^{\text {th }}$ percentile; center line, median; whiskers, full

755 data range in $\mathbf{d}$ and $\mathbf{e}$. The letters indicate the significance groups at $P<0.01$ (One-way ANOVA

756 and Tukey test) in $\mathbf{f}$ and $\mathbf{g}$. $P$ values (two-tailed, two-sample $t$-test) in $\mathbf{f}$ and $\mathbf{g}$. Exact sample sizes

757 (n) are shown in $\mathbf{f}$ and $\mathbf{g}$.

758

759

760

Supplementary Fig. 6. Transgenic complementation tests of tomato slclv3 single and slclv3 slcle9 double mutants.

762

a, b, Complementation tests of tomato slclv3 single mutants. Inflorescence images (a) and petal number quantifications (b) of WT and slclv3 compared to the $\mathrm{T}_{1}$ transgenic plants $g$ SlCLV3 ${ }^{\text {SlCLV } 3}$, $g P g C L E 9^{P g C L E 9}, g S l C L E 9^{P g C L E 9}, g S l C L V 3^{P g C L E 9}$, and $g S l C L V 3^{S l C L E 9}$. c, Diagrams of the constructs used for complementation tests of slclv3 slcle9 double mutants. gSlCLV3 ${ }^{\text {SlCLV3 }}$ (SlCLV3 genomic DNA). $g S l C L V 3^{S l C L E 9}$ (SlCLV3 genomic DNA including the sequence for SlCLE9 dodecapeptide). gSlCLE9 ${ }^{\text {SlCLE9 }}$ (SICLE9 genomic DNA). gSlCLE9SICLES6G (SICLE9 genomic DNA including the sequence for PgCLE9 dodecapeptide). Black and orange rectangles mark the coding sequences and the dodecapeptide sequences, respectively. The numbers with minus (-) and plus (+) signs indicate the positions of the upstream sequences and the downstream sequences from the adenines

771 compared to the $\mathrm{T}_{1}$ transgenic plants $g S l C L V 3^{S l C L V 3}-2$, gSlCLV3 $3^{\text {SICLE9 }}-2$, gSlCLE9SICLE9 -2 , and

772 gSlCLE9 ${ }^{\text {SICLE9S6G }}$. Data are based on at least three independent transgenic lines for each construct.

773 Box plots, $25^{\text {th }}-75^{\text {th }}$ percentile; center line, median; whiskers, full data range in $\mathbf{b}$ and $\mathbf{d}$. The letters

774 indicate the significance groups at $P<0.01$ (One-way ANOVA and Tukey test) in b and d. Exact 775 sample sizes (n) are shown in $\mathbf{b}$ and $\mathbf{d}$. 


\section{Supplementary Tables}

778 Supplementary Table 1. CLE dodecapeptide sequences of SICLV3 and S1CLE9 homologs

779 Supplementary Table 2. Differentially expressed genes between petunia WT and phclv3 from

780 mRNA-seq.

781 Supplementary Table 3. Differentially expressed genes between groundcherry WT and pgclv3

782 from mRNA-seq.

783 Supplementary Table 4. Primers used in this study.

786 Source data

787 Source data 1. CRISPR-generated mutations in this study

788 Source data 2. Raw data of relative expression for Supplementary Fig.1.

789 Source data 3. Quantification data for organ numbers in this study.

790 Source data 4. Quantification data for meristem size from Fig. 1, 2 and 3.

791 Source data 5. Normalized counts from mRNA-seq for Fig.2 and 3.

792 Source data 6. Syntenic region of SlCLV3 homologs, defined by Conservatory orthogroups

793 Source data 7. Syntenic region of SICLE9 homologs, defined by Conservatory orthogroups

794 Source data 8. Exact $P$-values in this study (one-way ANOVA with Tukey test)

\section{Supplementary Dataset}

798 Supplementary Dataset 1. Sequencing trace files.

799 Supplementary Dataset 2. Petunia PhCLE9 sequence.

800 Supplementary Dataset 3. CLV1 homolog sequences. 
bioRxiv preprint doi: https://doi.org/10.1101/2022.01.03.474791; this version posted January 3, 2022. The copyright holder for this preprint

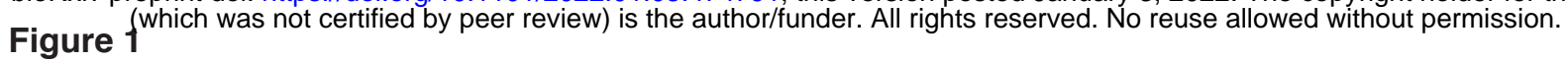

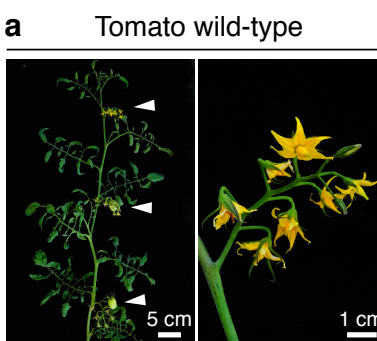

d

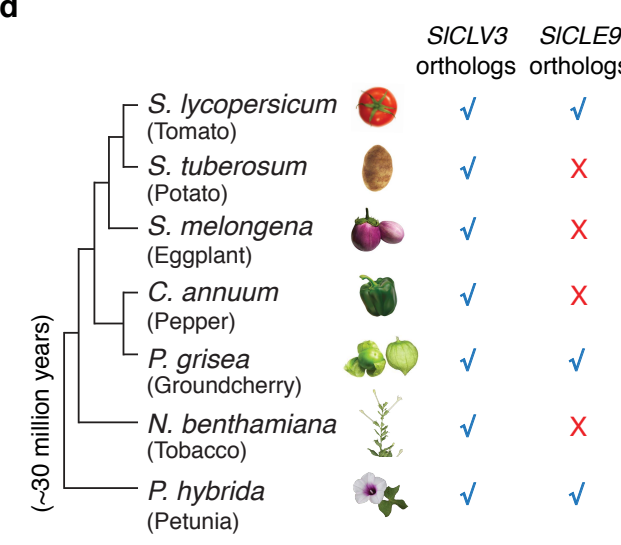

b Tomato s/c/v3

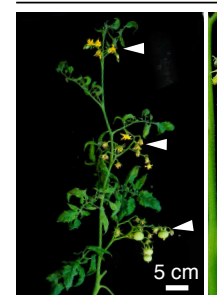

c

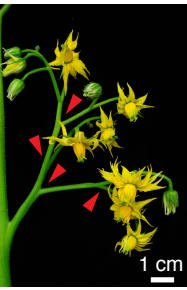

Tomato slclv3 slcle9

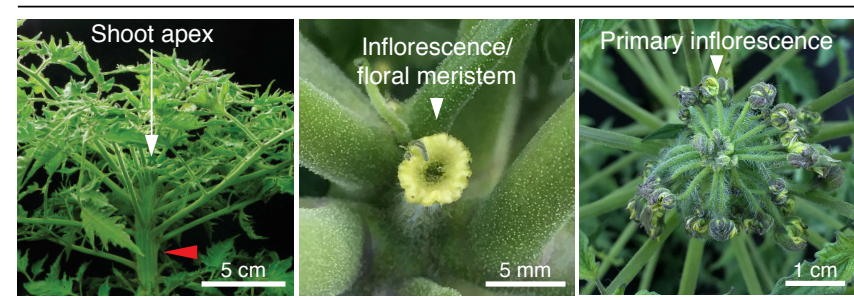

e

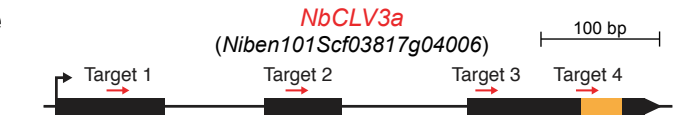

NbCLV3a AстCTTCCTGTTGCTGCCTGTTTTGGTTG(110) TAGCTCAAAATGCATTTCACAAAAGGAAG

NbCLV3a (151)TTAGGAAGCCAGTGGGTTGCTTTGGGAA (55) TGGGAGCTTAGGGGAATTCCAGCTGGTCC $n b c / v 3 a^{C R-1}$ ACTCTTCCTGTTGCTGCCTGTTTTTGGTTG (110) TAGCTCAAAATGCATTTCACAAAAGGAAG
(151) TTAGGAAGCCAGTGGGCTGC-------(55)----------$\begin{array}{ll}n b c / v 3 a^{C R-2} & \text { ACTCTTCCTGTTGCTGCCTGTTTTGGTTG }(110) \text { TAGCTCAAAATGCATTTCACAAAAGGAAG } \\ \text { (151) TTAGGAAGCCAGTGGGTGCTTTTGGGAA } 55) \text { TGGGAGCTTAGGGAATTC-AGCTGGTCC }\end{array}$

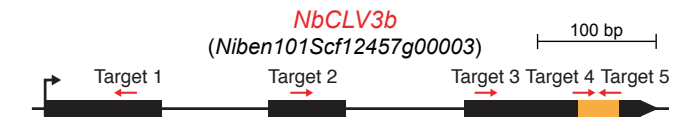

NbCLV3b TTCCTCTTGCTGTCTGTTTTGGTTGTT(110) AGCTCAAAATGCATTTCACAAAAGGAA(156) (42) GGGAGCTTAGGGGATCCCAGCTGGTCCTGATCCATTGCACCACAATGGTG $n b c / v 3 b^{C R-1}$ TTCCTCTITGCTGTCTGTTTTGGTTGTT (110) AGCTCAAAATGCATTTCACAAAAGGAA ( 156) nbC/V3b ${ }^{C R-1}$ CTGCATTTGGGAAGGGCT--------(42)----------AGCTGGTCTGATCCATTGCACCACAATGGTG $n b c / v 3 b^{C R-2}$ TTCCTCTTTGCTGTCTGTTTTGGTTGTT (110) AGCTCAAAATGCATTTCACAAAAGGAA (156) CTGCATTTGGGAAGGCTTTGCATGGAA ( 42 ) GGGAGCTTAGGG--------GCTGGTCCTGATC--TTGCACCACAATGGTG

$\mathbf{f}$

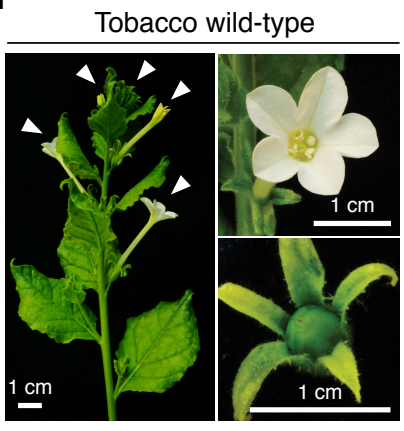

h

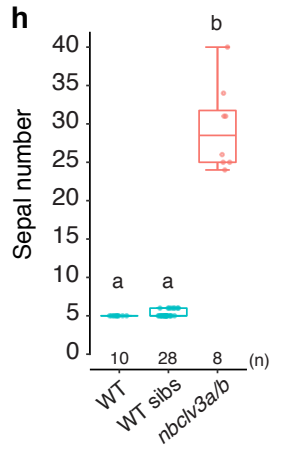

g

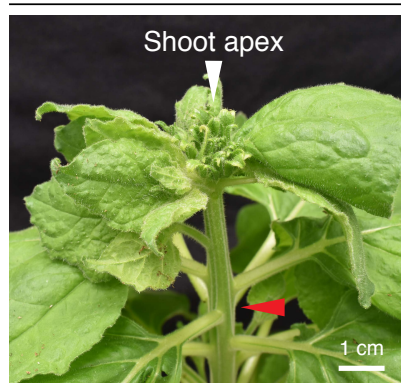

j

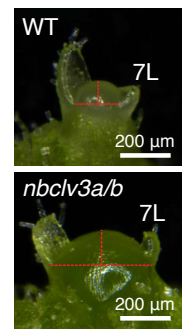

$\mathbf{k}$
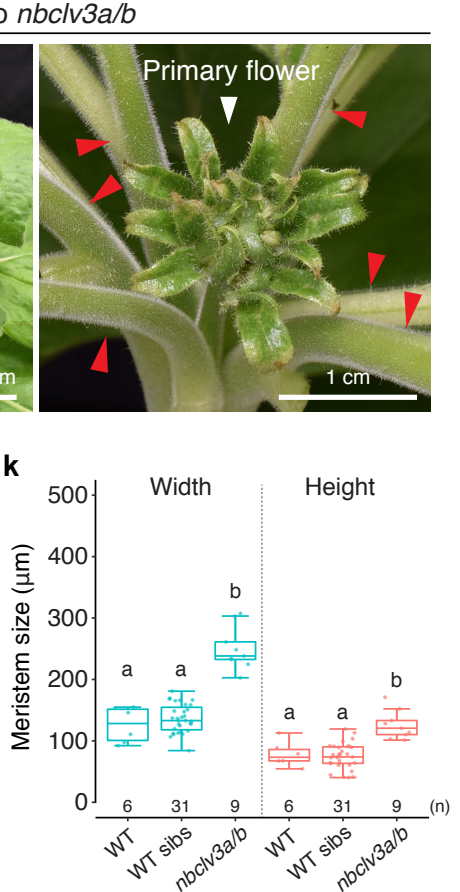

Fig. 1. Loss of the tobacco SICLE9 ortholog abolished compensation.

a, Shoot and inflorescence of tomato wild-type (WT). White arrowheads, inflorescences. b, Shoot and inflorescence of tomato slclv3. White arrowheads, inflorescences; red arrowheads, branches. c, Side and top-down view of tomato slclv3 slcle 9 shoot, inflorescence/floral meristem, and primary inflorescence. The red arrowhead indicates a fasciated shoot stem. d, Presence-absence variation of SlCLE9 orthologs in the Solanaceae. Phylogenic relationships of the indicated species are shown. The blue checkmarks and the red Xs indicate presence and absence of the orthologs, respectively. e, Gene structures, and CRISPR-generated mutations of $N b C L V 3 a$ and $N b C L V 3 b$. Orange rectangles indicate the CLE dodecapeptides regions. Targeted guide RNA and protospacer-adjacent motif (PAM) sequences are highlighted in red and bold underlined, respectively. Blue letters and dashes indicate insertions and deletions, respectively. Numbers in parentheses represent gap lengths. DNA sequences of gRNA target site 2 for both $N b C L V 3 a$ and $N b C L V 3 b$ are identical. f, Shoot, flower, and fruit pod of tobacco WT. White arrowheads, flowers. g, Side and top-down views of 
$n b c l v 3 a / b$ null mutants showing the shoot and primary flower. Red arrowheads indicate fasciated primary shoot (left panel) and shoot branches (right panel). h, Sepal number of primary flower from tobacco WT, WT sibling plants (WT sibs) and $n b c l v 3 a / b$ plants. i, Branch number of WT, WT sibs and $n b \operatorname{clv} 3 a / b$. j, Primary shoot apical meristems from WT and $n b \operatorname{lv} 3 a / b$. Red dotted lines mark width and height for meristem size quantification. 7L, 7th leaf primordium. k, Quantification of meristem width and height from WT, WT sibs and $n b c l v 3 a / b$. Box plots, $25^{\text {th- }} 75^{\text {th }}$ percentile; center line, median; whiskers, full data range in $\mathbf{h}, \mathbf{i}$ and $\mathbf{k}$. Exact sample sizes (n) for replicate types are indicated in $\mathbf{h}, \mathbf{i}$ and $\mathbf{k}$. Letters indicate significance groups at $P<0.01$ (One-way ANOVA and Tukey test) in $\mathbf{h}, \mathbf{i}$ and $\mathbf{k}$. WT sibs are a mix of $n b c l v 3 b$ and $n b c l v 3 a /+n b c l v 3 b$ genotypes, which show wild-type phenotypes in $\mathbf{h}, \mathbf{i}$ and $\mathbf{k}$. See Source Data 4. 


\section{Figure 2}

a

PhCLV3

(Peaxi162Scf00429g00081)

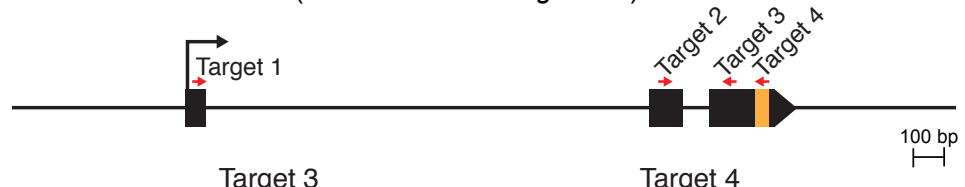

WT AGCCAATGGGCTGCTTATGGAAAGGGCTTA (64) TTCCAGCTGGTCCTGATCCAATTCACCATCATGGTGG

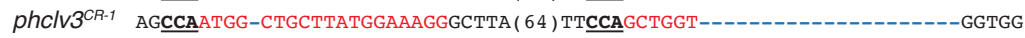
phc/v3 $3^{\text {CR-2 }}$ AGCCAATGG----CTTATGGAAAGGGCTTA $(64)$ TTCCAGCTGGTCCTGATCCAATTCACCATCATGGTGG
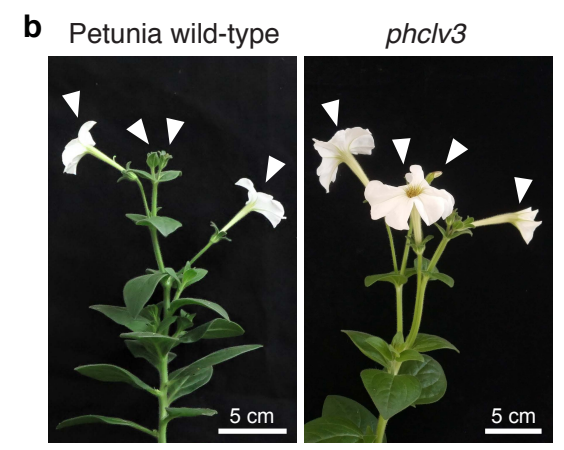

C
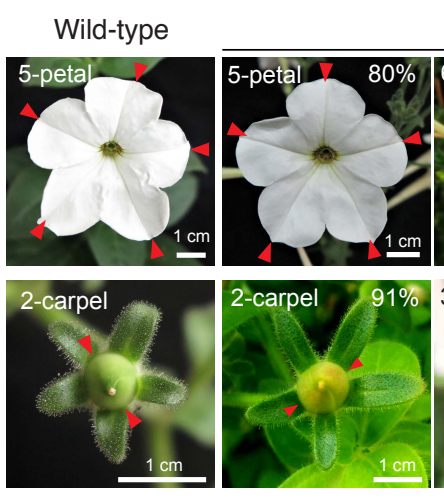

phclv3
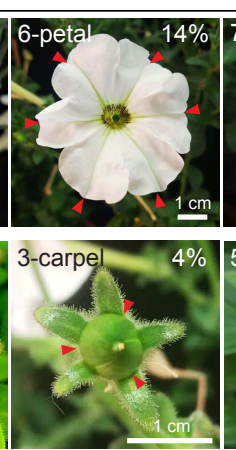

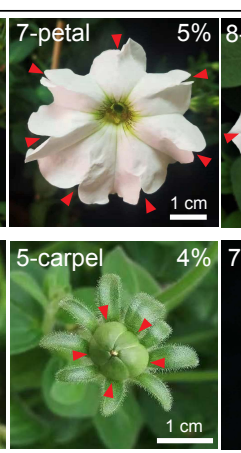

4\% 7 -carpel

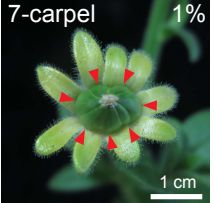

d

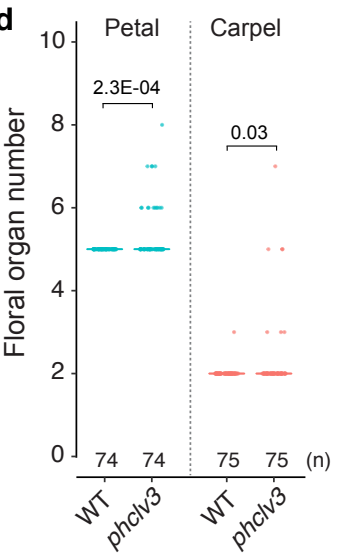

e

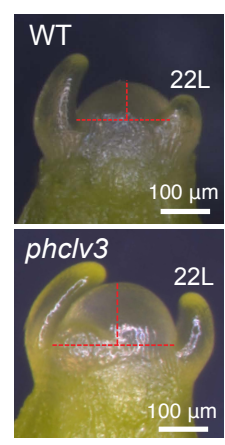

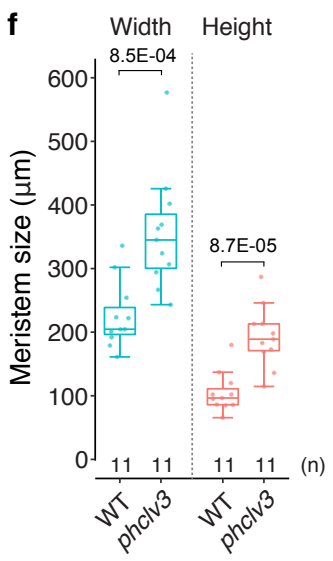

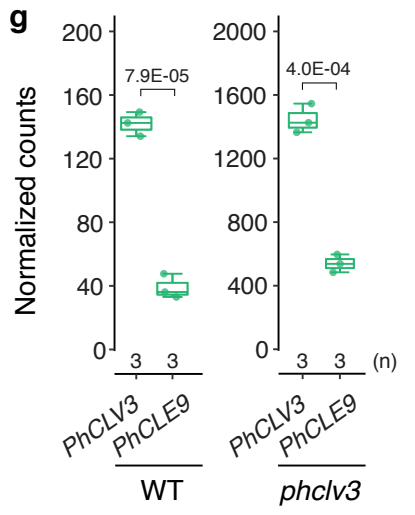

h

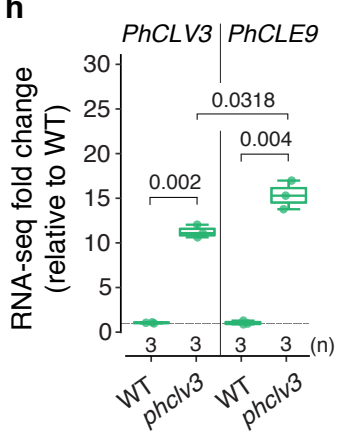

\section{Fig. 2. Weak fasciation of phclv3 mutants in petunia indicates more potent compensation.}

a, Gene structure and sequences of two phclv3 null alleles. Guide RNA and PAM sequences are highlighted in red and bold underlined, respectively. The orange rectangles in the gene structures represent the regions for CLE dodecapeptides. Numbers in parentheses represent gap lengths. Blue dashes indicate deletions. b, Shoot of petunia WT and phclv3 plants. White arrowheads, flowers. c, Representative flowers and fruit pods of petunia WT and phclv3 plants. Red arrowheads mark petals or carpels. Percentages indicate the proportions of flower and pod phenotypes. d, Quantification of petal and carpel numbers of WT and phclv3. e, Primary shoot apical meristems from petunia WT and phclv3. Red dotted lines mark width and height for meristem size quantification. 22L, 22 th leaf primordium. f, Quantification of meristem width and height from petunia WT and phclv3. g, Normalized read counts of PhCLV3 and PhCLE9 from WT and phclv3 meristems. Expression unit, fragments per kilobase of transcript per million (FPKM). h, Expression foldchange of PhCLV3 and PhCLE9 relative to the normalized counts of WT from phclv3. Box plots, $25^{\text {th- }} 75^{\text {th }}$ percentile; center line, median; whiskers, full data range in $\mathbf{d}, \mathbf{f}, \mathbf{g}$ and $\mathbf{h}$. $P$ values (two-tailed, two-sample $t$-test) in $\mathbf{d}, \mathbf{f}, \mathbf{g}$ and $\mathbf{h}$. Exact sample sizes (n) are shown as discrete numbers in $\mathbf{d}, \mathbf{f}, \mathbf{g}$ and $\mathbf{h}$. Each replicate (n) is from 50-60 meristems in $\mathbf{g}$ and $\mathbf{h}$. 
bioRxiv preprint doi: https://doi.org/10.1101/2022.01.03.474791; this version posted January 3, 2022. The copyright holder for this preprint Figure 3 (which was not certified by peer review) is the author/funder. All rights reserved. No reuse allowed without permission.

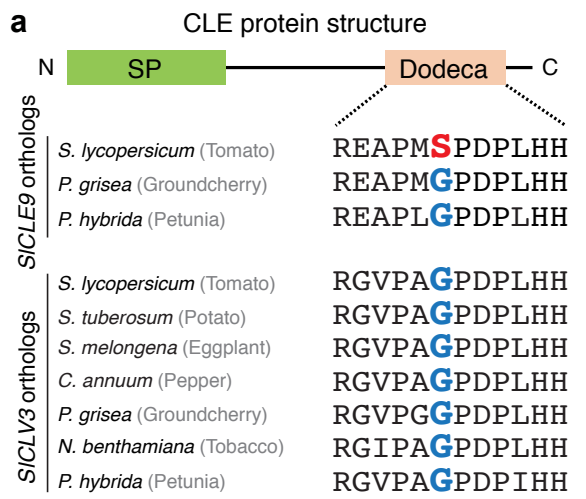

b
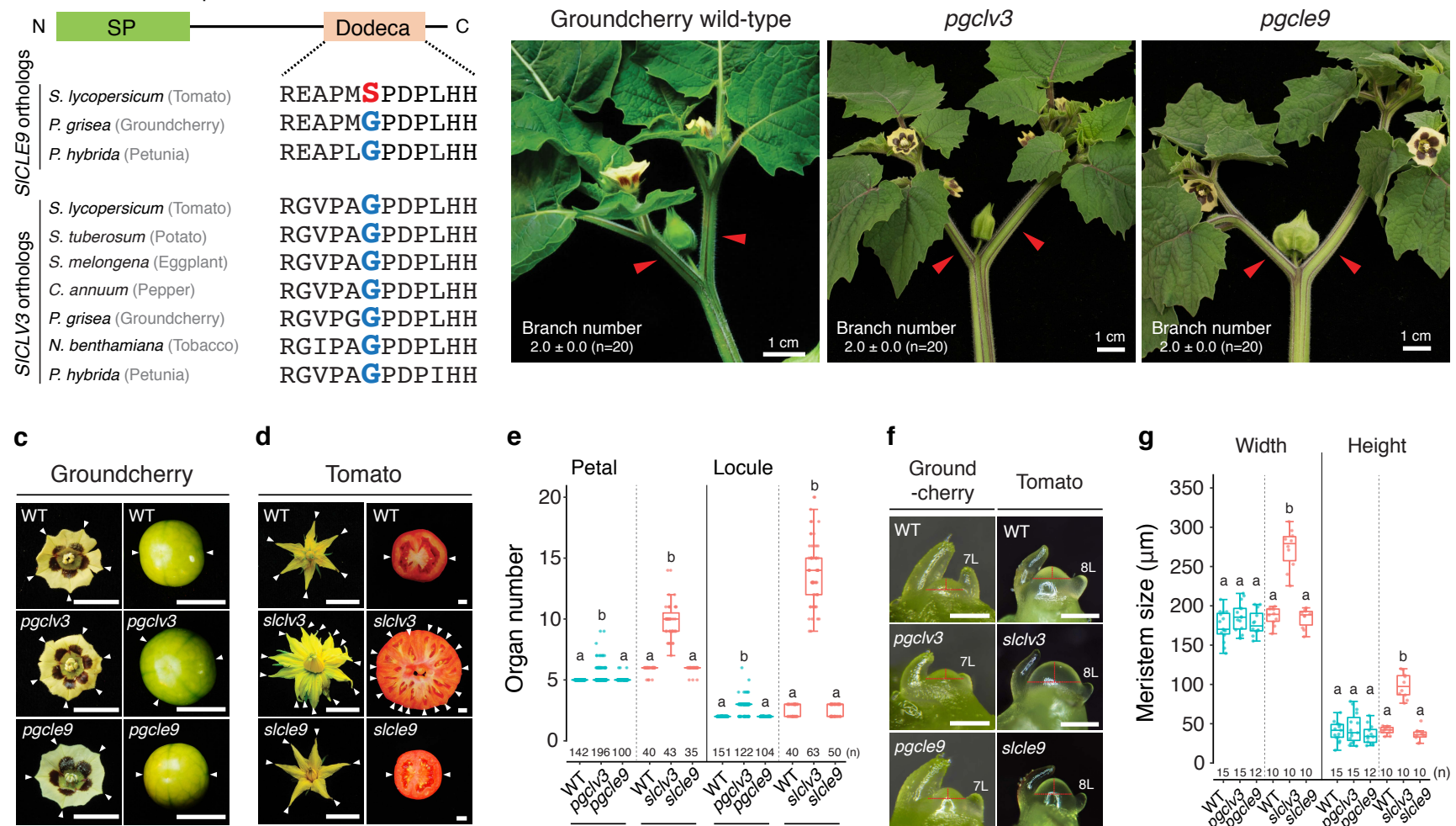

d

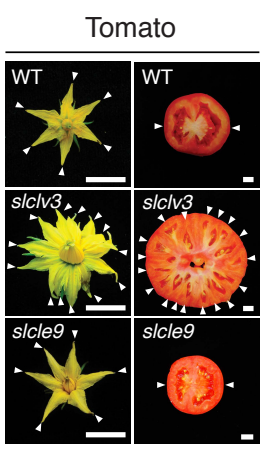

e
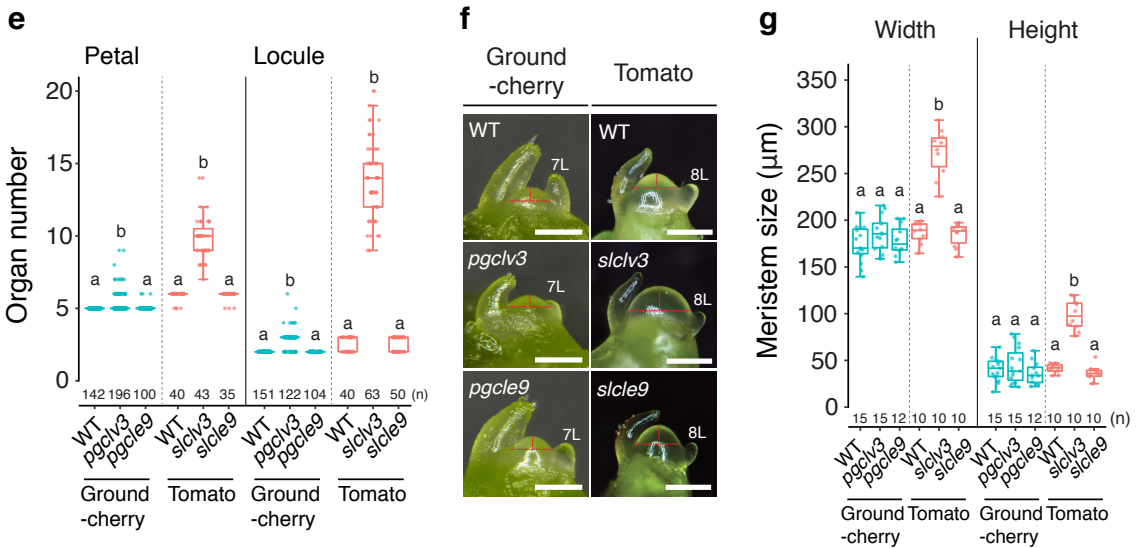

h

Tomato

Groundcherry
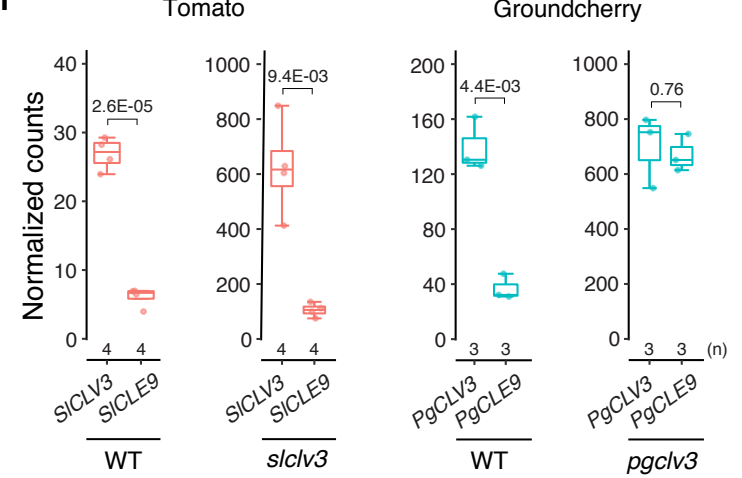

i

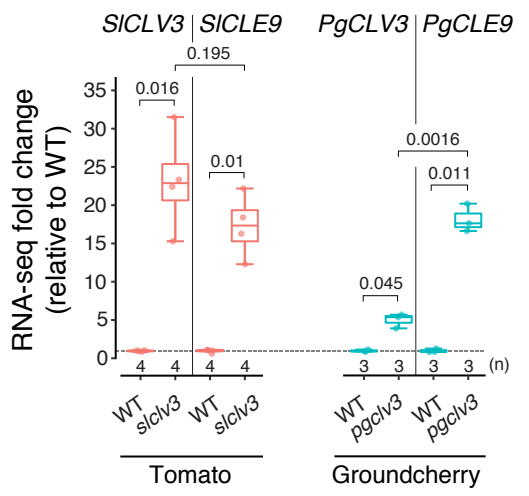

j

Groundcherry pgclv3 pgcle9
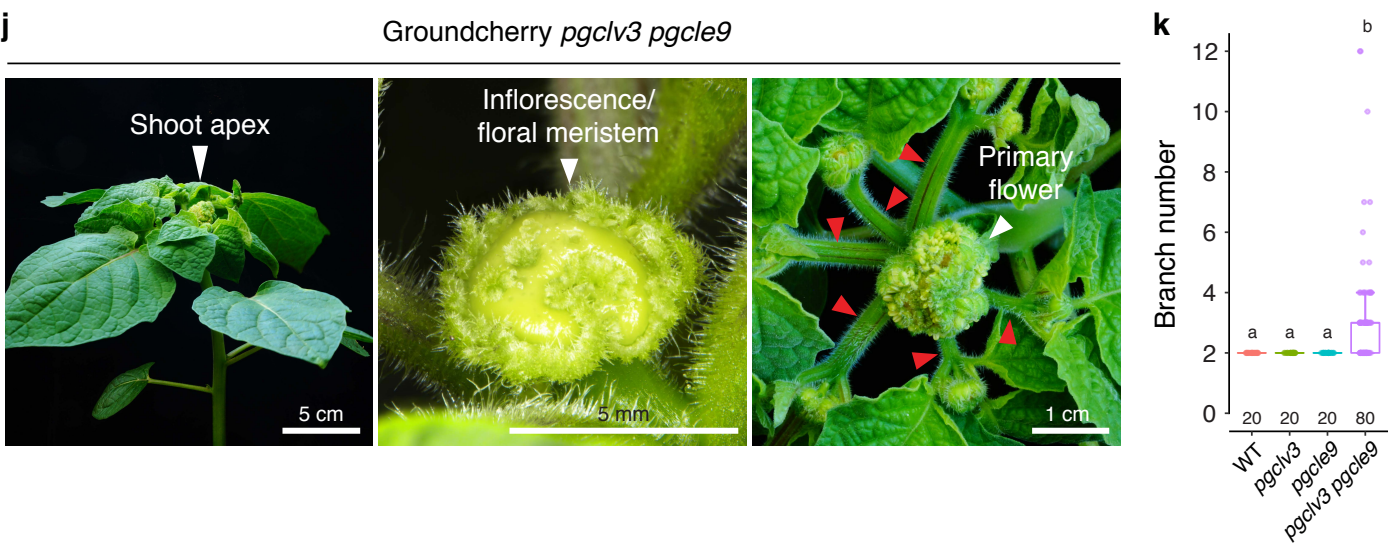

Fig. 3. A highly conserved dodecapeptide amino acid is associated with potent compensation in groundcherry.

a, CLE protein structure and dodecapeptide sequences of SICLE9 and SICLV3 orthologs in the Solanaceae. The sixth residue of the dodecapeptides are highlighted by red or blue bold font. b, Shoot and inflorescences of groundcherry WT, pgclv3 and pgcle 9 plants. Red arrowheads mark two side shoots that develop after single-flowered inflorescences. c, Representative flowers and fruits from groundcherry WT, pgclv3, and pgcle 9 plants. Scale bar, 1 $\mathrm{cm}$. d, Representative flowers and fruits from tomato WT, slclv3, and slcle9 plants. White arrowheads mark petals or locules. Scale bar, $1 \mathrm{~cm}$. 
e, Quantification and comparison of petal and locule numbers from groundcherry WT, pgclv3, pgcle9 and tomato WT, slclv3, and slcle9 plants. f, Primary shoot apical meristems from groundcherry WT, pgclv3, pgcle 9 and tomato WT, slclv3, and slcle 9 plants. 7L, 8L: $7^{\text {th }}$ and $8^{\text {th }}$ leaf primordia, respectively. Red dotted lines indicate width and height for meristem size measurements, Scale bar, $200 \mu \mathrm{m}$. g, Quantification of meristem width and height from groundcherry WT, pgclv3, pgcle9, tomato WT, slclv3, and slcle9 plants. h, Normalized RNA-seq read counts of SlCLV3, SlCLE9, $P g C L V 3$, and $P g C L E 9$ from tomato WT, slclv3, groundcherry WT and pgclv3 meristems. Expression units are counts per million (CPM) for tomato and transcripts per million (TPM) for groundcherry i, Expression fold-change of SlCLV3, SlCLE9, PgCLV3, and PgCLE9 relative to the normalized counts of WT expressions of these genes in the indicated genotypes. $\mathbf{j}$, Side and top-down views of a pgclv3 pgcle 9 double mutant shoot, inflorescence/floral meristem, and primary flower. Red arrowheads indicate branches that emerged after the primary flower. k, Branch number of WT, pgclv3, pgcle9, and pgclv3 pgcle9 plants. Box plots, 25th-75th percentile; center line, median; whiskers, full data range in e, $\mathbf{g}$, h, i and $\mathbf{k}$. The letters indicate the significance groups at $P<0.01$ (One-way ANOVA and Tukey test) in $\mathbf{e}, \mathbf{g}$ and $\mathbf{k} . P$ values (two-tailed, two-sample $t$-test) in $\mathbf{h}$ and $\mathbf{i}$. Exact sample sizes (n) are shown in $\mathbf{e}, \mathbf{g}, \mathbf{h}, \mathbf{i}$ and $\mathbf{k}$. Each replicate (n) is from 30-35 meristems in $\mathbf{h}$ and $\mathbf{i}$. 
bioRxiv preprint doi: https://doi.org/10.1101/2022.01.03.474791; this version posted January 3, 2022. The copyright holder for this preprint (which was not certified by peer review) is the author/funder. All rights reserved. No reuse allowed without permission.

Figure 4

a

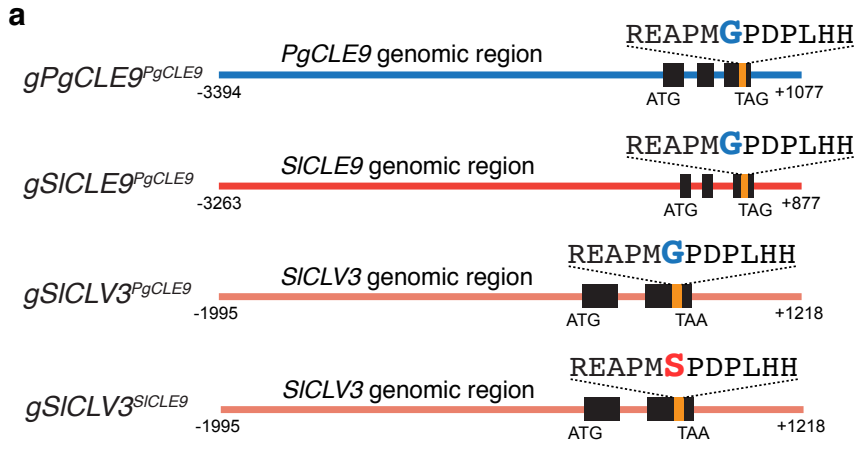

b

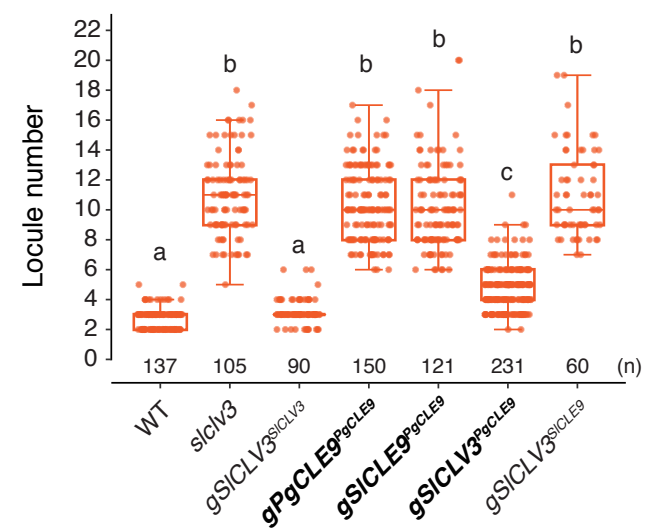

C

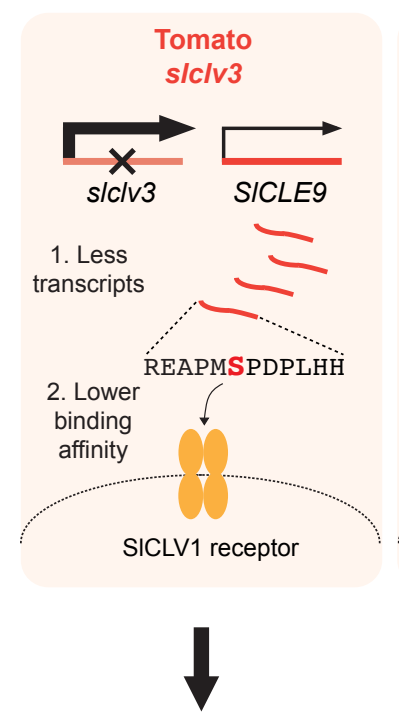

Weak compensation

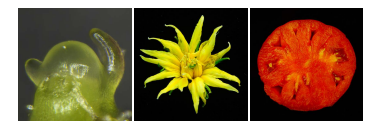

Strong fasciation

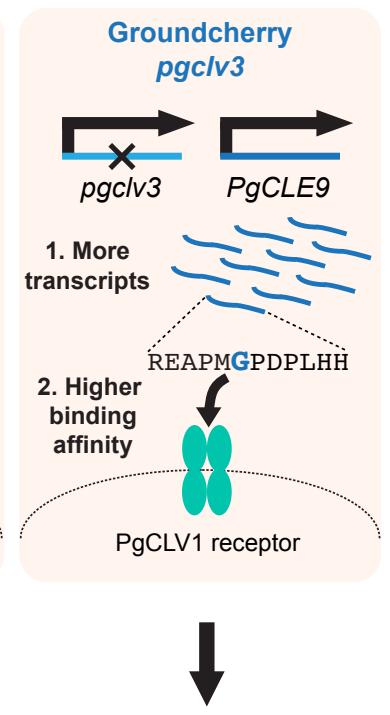

Strong compensation

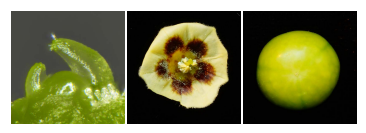

Weak fasciation

\section{Fig. 4. Variation in Solanaceae compensation is due to changes in both the SICLE9 ortholog dodecapeptide and its expression.}

a, Diagrams of constructs used for complementation tests. gPgCLE9PgCLE9 (PgCLE9 genomic DNA). gSlCLE9PgCLE9 (SlCLE9 genomic DNA including the sequence for PgCLE9 dodecapeptide). gSlCLV3PgCLE9 (SlCLV3 genomic DNA including the sequence for PgCLE9 dodecapeptide). gSlCLV3SlCLE9 (SlCLV3 genomic DNA including the sequence for SICLE9 dodecapeptide). Black and orange rectangles mark the coding sequences and the dodecapeptide sequences, respectively. The numbers with minus (-) and plus $(+)$ signs indicate the positions of the upstream sequences and the downstream sequences from the adenines of start codons, respectively. b, Locule number quantification from WT and slclv3 mutants compared to $\mathrm{T}_{1}$

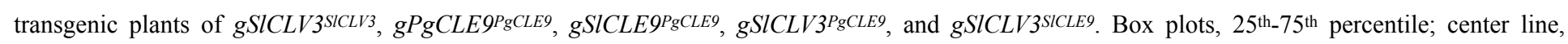
median; whiskers, full data range. The letters indicate the significance groups at $P<0.01$ (One-way ANOVA and Tukey test). Exact sample sizes (n) are shown as discrete numbers. Data are based on at least 10 independent transgenic lines for each construct. c, A proposed model for differences in active compensation between tomato and groundcherry. The more potent active compensation in groundcherry compared to tomato is due to both the glycinecontaining PgCLE9 dodecapeptide and its higher expression. 


\section{Figure 5}

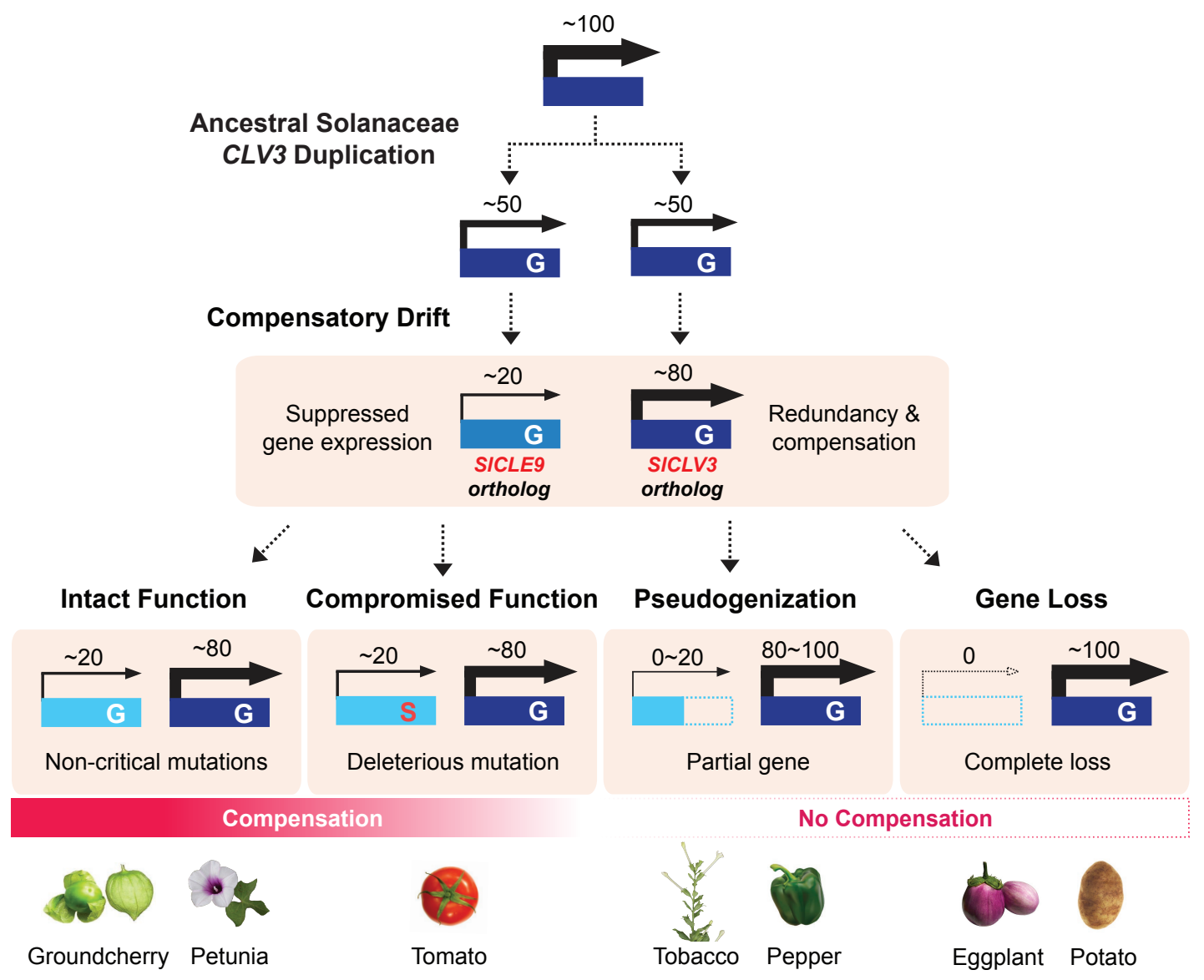

Fig. 5. Summary and model of the dynamic evolution of SICLV3 and SICLE9 orthologs and their compensation relationships in the Solanaceae. Dark blue, blue, and sky blue rectangles indicate the coding region of the genes. Arrows and their thickness represent gene expressions and their relative levels, respectively. Numbers above the arrows indicate hypothetical relative proportions of SlCLV3 and SlCLE9 ortholog expression levels. 'G' and 'S' within the rectangles denote the sixth amino acid of each CLE dodecapeptide. Dashed rectangles mark deletions of the coding region, resulting in pseudogenes (pepper and tobacco) and complete gene loss (eggplant, potato) in each genome. The red gradient bar reflects the loss of active compensation and its degree, depending on the indicated genetic variation. 
bioRxiv preprint doi: https://doi.org/10.1101/2022.01.03.474791; this version posted January 3, 2022. The copyright holder for this preprint (which was not certified by peer review) is the author/funder. All rights reserved. No reuse allowed without permission.

\section{Supplementary Fig. 1}

a

NbCLV3a AстCTTCCTGTTGCTGCCTGTTTTGGTTG (110) TAGCTCAAAATGCATTTCACAAAAGGAAG (151) TTAGGAAGCCAGTGGGCTGCTTTTGGGAA (55) TGGGAGCTTAGGGGAATTCCAGCTGGTCC

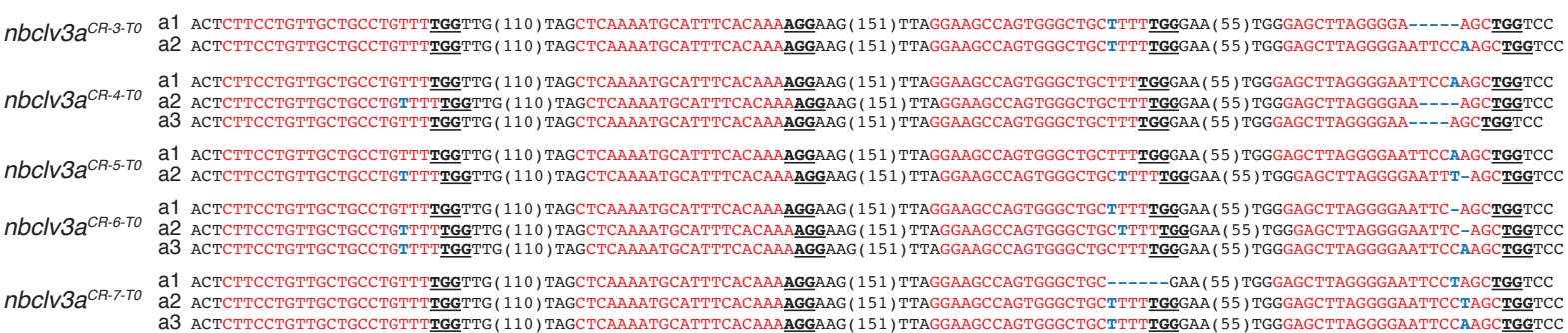

b

NbCLV3b TTCCTCTTGCTGTCTGTTTTGGTTGTT (110) AGCTCAAAATGCATTTCACAAAAGGAA (156) CTGCATTTGGGAAGGGTTGCATGGAA (42) GGGAGCTTAGGGGAATCCCAGCTGGTCCTGATCCATTGCACCACAATGGTG

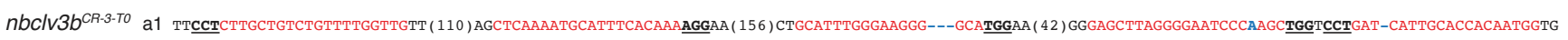
$n b c / v 3 b^{C R-4-T O}$ a1 TTCCTCTTTGCTGTCTGTTTTGGTTGTT (110)AGCTCAAAATGCATTTCACAAAAGGAA (156) CTGCATTTGGGAAGGGCTTTGCATGGAA ( 42 ) GGGAGCTTAGGGGAATCCCAAGCTGGTCCTGAT-CATTGCACCACAATGGTG $\begin{array}{lll}n b c / v 3 b & \text { ar-5-To } \\ \text { a2 TTCCTCTTGCTGTCTGTTTTGGTTGTT (110) AGCTCAAAATGCATTTCACAAAAGGAA (156) CTGCATTTGGGAAGGGCTTTGCATGGAA (42) GGGAGCTTAGGGG------AGCTGGTCCTGATCCATTGCACCACAATGGTG }\end{array}$

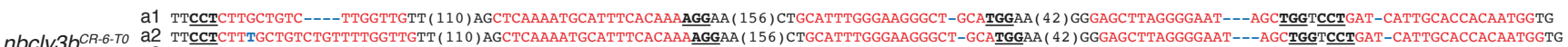
a3 TTCCTCTTGCTGTCTGTTTTGGTTGTT (110) AGCTCAAAATGCATTTCACAAAAGGAA (156) CTGCATTTGGGA-----TGCATGGAA (42) GGGAGCTTAGGGGAATC--AGCTGGTCCTGAT-CATTGCACCACAATGGTG a4 TTCCTCTTG----CTGTTTTGGTTGTT (110) AGCTCAAAATGCATTTCACAAA

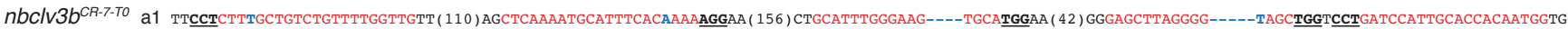

C

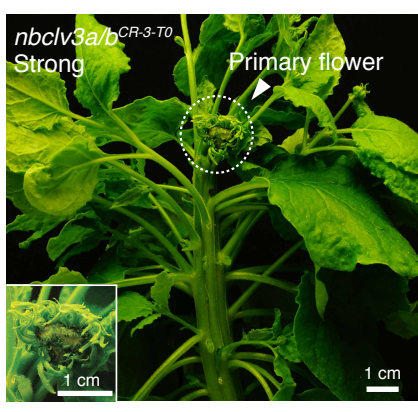

d
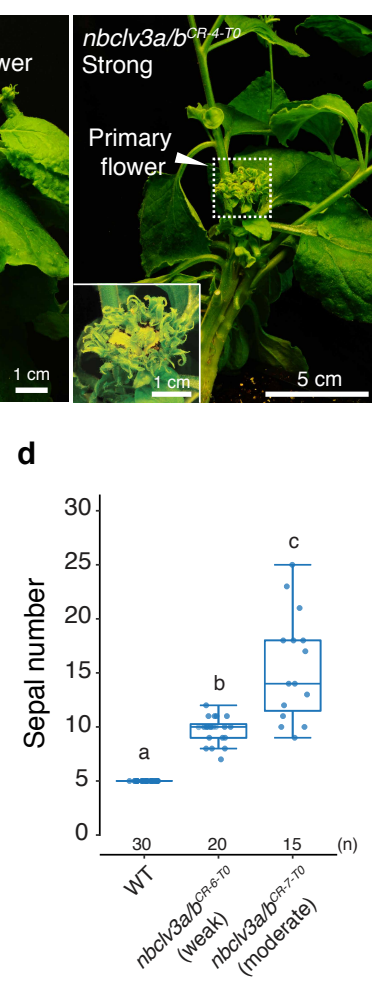

d
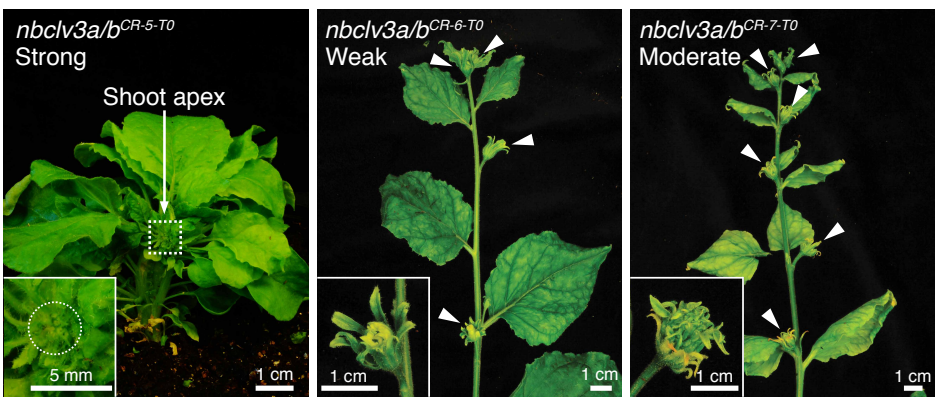

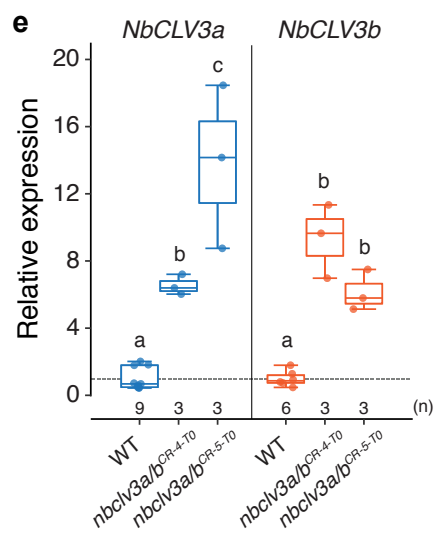

\section{Supplementary Fig. 1. CRISPR-generated mutations of the tobacco $N b C L V 3 a$ and $N b C L V 3 b$ genes.}

a, CRISPR-generated sequences of $n b c l v 3 a$ mutant alleles. b, CRISPR-generated sequences of $n b c l v 3 b$ mutant alleles. Guide RNA and PAM sequences are highlighted in red and bold underlined, respectively. Blue letters and dashes indicate insertions and deletions, respectively. Numbers in parentheses represent gap lengths. c, Shoots and inflorescences of $n b c l v 3 a / b \mathrm{~T}_{0}$ plants. Three strong lines $\left(n b c l v 3 a / b^{C R-3-T 0}, n b c l v 3 a / b C R-4-T 0\right.$ and $\left.n b c l v 3 a / b^{C R-5-T O}\right)$ show similar phenotypes compared to null $n b c l v 3 a / b$ mutants in Fig. 1g. Weak $(n b c l v 3 a / b C R-6-T 0)$ and moderate $\left(n b c l v 3 a / b^{C R-7-T O}\right)$ lines show regular shoot architecture but fasciated floral organs. White arrowheads indicate flowers. d, Sepal number of weak and moderate $n b c l v 3 a / b \mathrm{~T}_{0}$ plants. e, Relative expressions of $N b C L V 3 a$ and $N b C L V 3 b$ in shoot apices from $n b c l v 3 a / b \mathrm{~T}_{0}$ chimeric mutants, normalized to $N b P P 2 A$. Dashed line, value ' 1 ' on the y-axis. Three and one biological replicates for WT and $n b c l v 3 a / b$ plants, respectively; two or three technical replicates included. Box plots, $25^{\text {th }}-75^{\text {th }}$ percentile; center line, median; whiskers, full data range in $\mathbf{d}$ and $\mathbf{e}$. The letters indicate the significance groups at $P<0.01$ (One-way ANOVA and Tukey test) in $\mathbf{d}$ and $\mathbf{e}$. The exact sample sizes (n) are shown as discrete numbers in $\mathbf{d}$ and $\mathbf{e}$. 
bioRxiv preprint doi: https://doi.org/10.1101/2022.01.03.474791; this version posted January 3, 2022. The copyright holder for this preprint (which was not certified by peer review) is the author/funder. All rights reserved. No reuse allowed without permission.

\section{Supplementary Fig. 2}

a

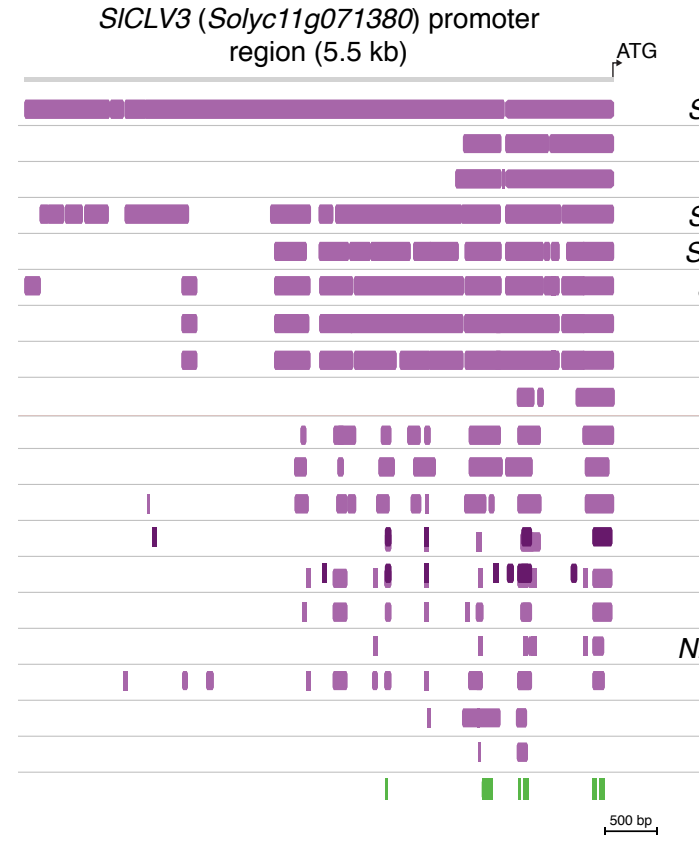

SICLE9 (Solyc06g074060) promoter

Solanum pimpinellifolium Solanum chilense Solanum pennellii Solanum lycopersicoides Solanum appendiculatum Solanum pinnatisectum Solanum chacoense Solanum tuberosum Solanum melongena Capsicum annuum Jaltomata sinuosa Physlais grisea

Nicotiana benthamiana Nicotiana tabacum Nicotiana attenuata Nicotiana tomentosiformis Nicotiana obtusifolia Petunia inflata Petunia axillaris

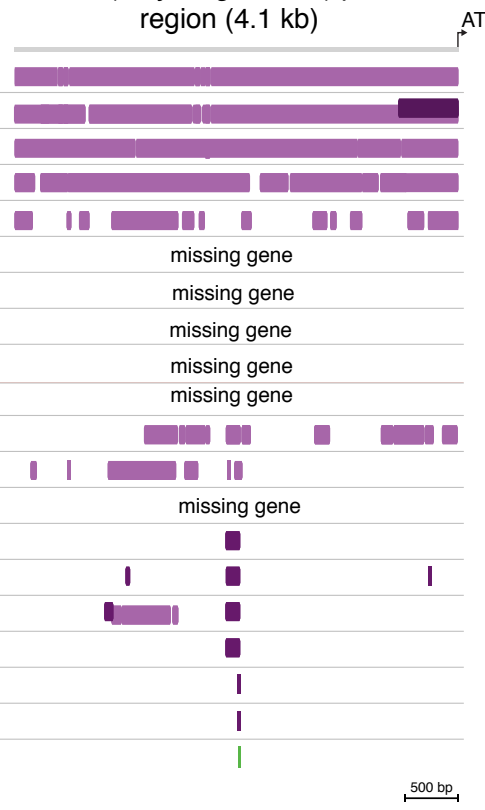

b CLV3 dodecapeptide Consensus
Identity

S. lycopersicum

S. pimpinellifolium

S. arcanum

S. chilense

S. habrochaites

S. pennellii

S. lycopersicoides

S. appendiculatum

S. pinnatisectum

S. tuberosum

S. chacoense

S. melongena

C. annuum

J. sinuosa

P. grisea

$N$. benthamiana

N. tabacum

N. attenuata

$N$. tomentosiformis

N. obtusifolia

$P$. inflata

$P$. axillaris
C

\section{CLE9 dodecapeptide}

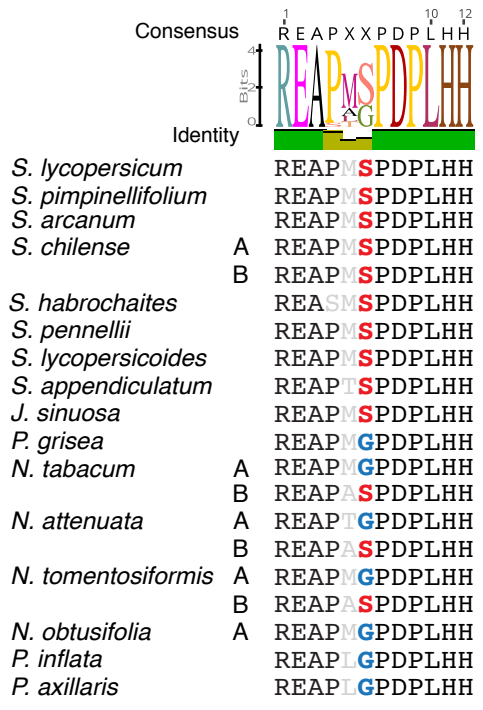

Supplementary Fig. 2. Conserved noncoding sequence (CNS) analysis of the promoter regions of SICLV3 and SlCLE9 orthologs in the Solanaceae family.

a, Conservatory analysis of Solanaceae CLV3 and CLE9 promoters. Purple boxes define highly similar regions of each gene's orthologs in the indicated species, and dark purple boxes define highly similar regions of the paralogous gene (e.g. $C L V 3 B$ or $C L E 9 B$ ) in the indicated species. Green boxes define Solanaceae CNSs. b, Multiple alignment and logo sequences of SLCLV3 dodecapeptide orthologs in the Solanaceae family. c, Multiple alignment and logo sequences of SICLE9 dodecapeptide orthologs in the Solanaceae family. 
bioRxiv preprint doi: https://doi.org/10.1101/2022.01.03.474791; this version posted January 3, 2022. The copyright holder for this preprint (which was not certified by peer review) is the author/funder. All rights reserved. No reuse allowed without permission.

\section{Supplementary Fig. 3}

a

b

C

\section{d}
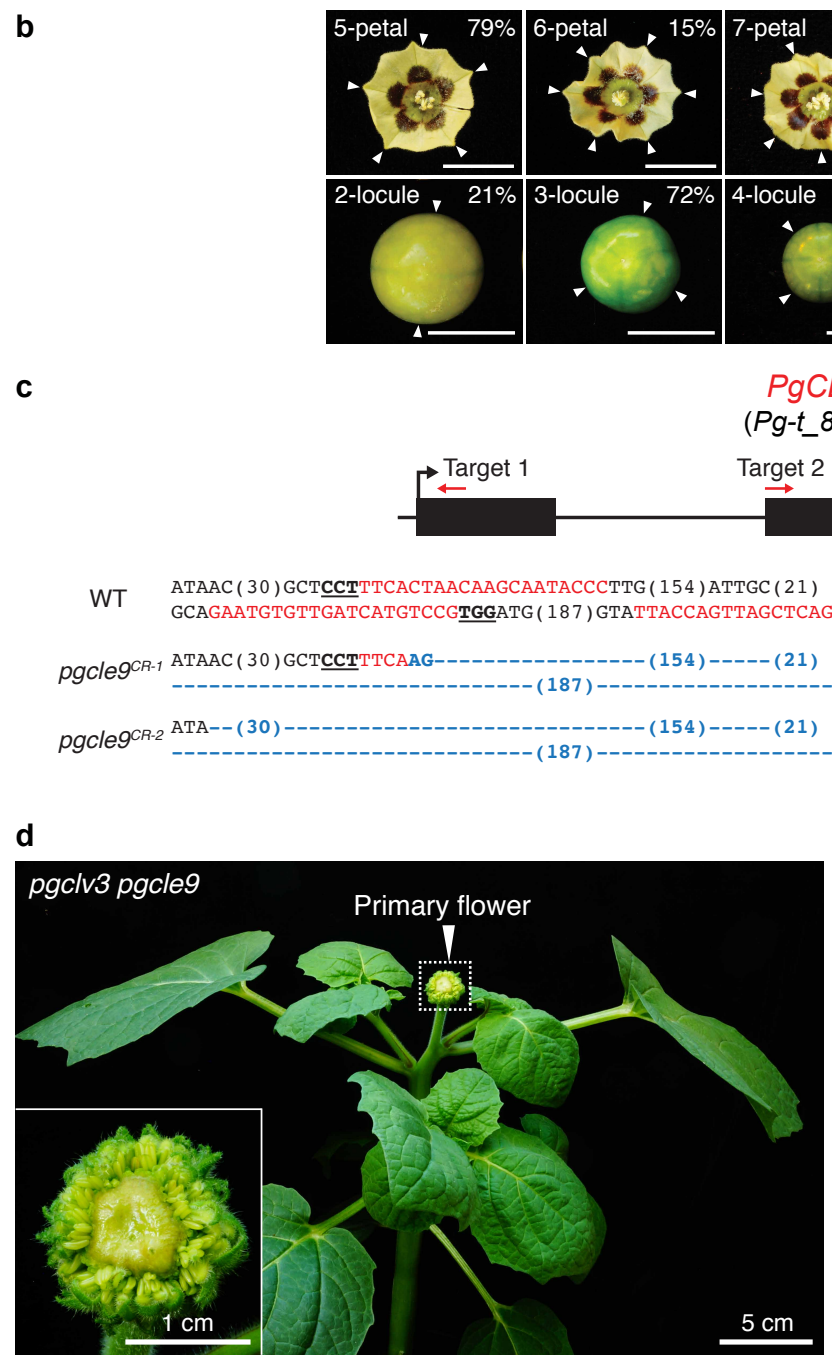

WT ATAAC (30)GCTCCTTTCACTAACAAGCAATACCCTTG(154)ATTGC (21)

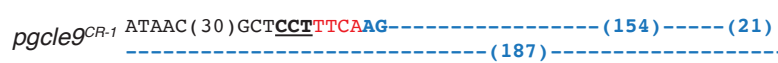

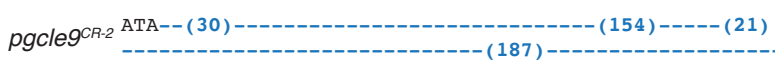

PgCLV3

(Pg-t_111)

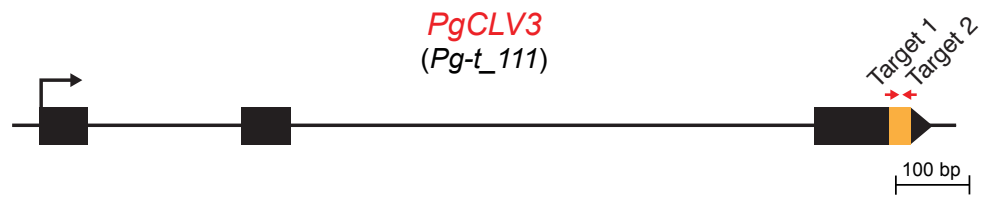

WT TGGGAGCTAAGAGGAGTTCCAGGTGGTCCTGATCCATTGCATCACAATGGTGTTAATCC pgclv $3^{\text {CR-1 }}$ TGGGAGCTAAGAGGAGTTCCCAGGTGTCCTGATCCATTGCATCACAATGGTGTTAATCC pgc/V3 $3^{\text {CR-2 }}$ TGGGAGCTAAGAGGAG----AGGTGGTCCTGATCCATTGCATCACAATGGTGTTAATCC
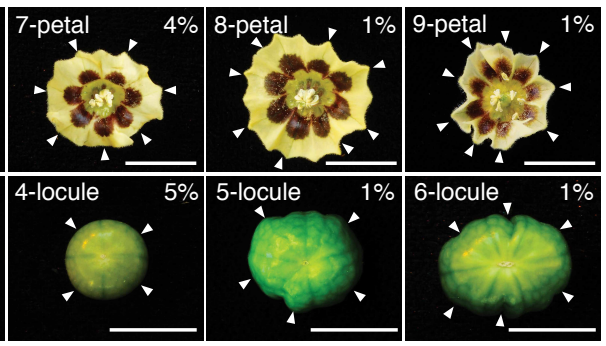

PgCLE9

(Pg-t_84762)

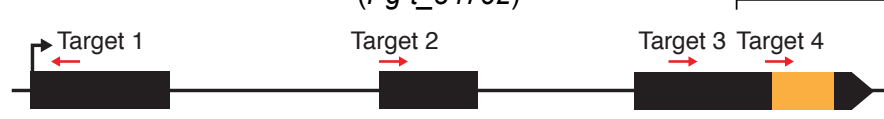

GCAGAATGTGTTGATCATGTCCGTGGATG ( 187 ) GTATTACCAGTTAGCTCAGAGAATGGGAA ( 19 ) GTAGAATTAAGGGAAGCACCAATGGGACC ( 20 ) GCAAT

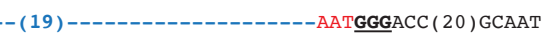

GACC $(20)$ GCAAT

e
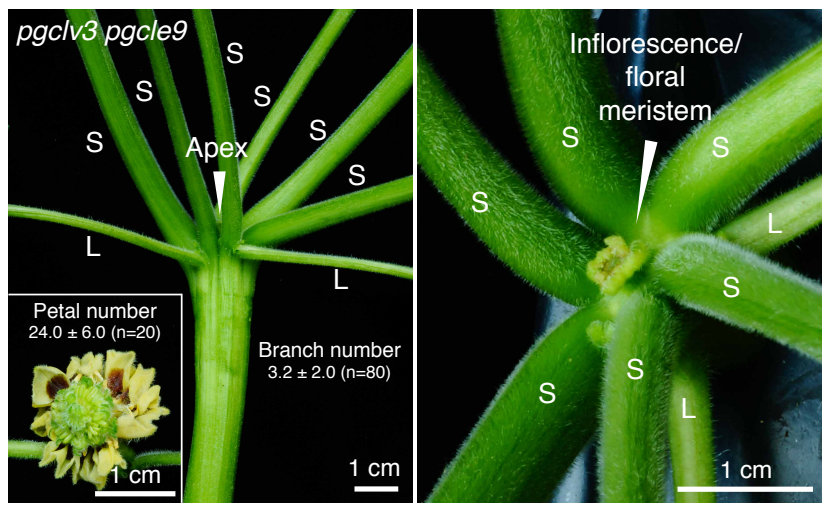

Supplementary Fig. 3. CRISPR-generated mutations of groundcherry PgCLV3 and PgCLE9.

a, Gene structure and sequences of pgclv3 CRISPR mutants. b, Flowers and fruits of pgclv3. White arrowheads mark petals or locules. Percentages indicate the proportions of flower and fruit phenotypes. Scale bar, $1 \mathrm{~cm}$. c, Gene structure and sequences of pgcle 9 CRISPR mutants. The orange rectangles in the gene structures indicate the regions of the CLE dodecapeptides in a and c. Guide RNA and PAM sequences are highlighted in red and bold underlined, respectively, in a and c. Blue letters and dashes indicate insertions and deletions, respectively, in a and c. Numbers in parentheses represent gap lengths in a and c. d, Shoot and an extremely fasciated primary flower of the pgclv3 pgcle9 double mutant. e, Development of multiple extra shoots (S) from the primary shoot and apex of a pgclv3 pgcle9 double mutant. L, leaf petioles. 
bioRxiv preprint doi: https://doi.org/10.1101/2022.01.03.474791; this version posted January 3, 2022. The copyright holder for this preprint (which was not certified by peer review) is the author/funder. All rights reserved. No reuse allowed without permission.

\section{Supplementary Fig. 4}

a SICLV1

StCLV1

SmCLV1

CaCLV1

PgCLV1

NbCLV1

PhCLV1
PSEFGNISTLKLLDLGNCNLDGEVPPSLGNLKKLHSLFLQVNRLTGHIPSELSGLESLMS 293 PSEFGNISTLKLLDLGNCNLDGEVPPSLGNLKKLHTLFLQVNRLTGRIPSELSGLESLMS 292 PSEFGSISTLKLLDLGNCNLEGEIPPSLGNLKKLHTLFLQMNRLTGHIPTELSGLESLMS 292 PPEFGNISTLKLLDLGSCNLDGEIPPSLANLKKLHSLFLOMNRLTGRIPSELSGLYSLMS 295 PPEFGSISTLKYLDLGSCNLDGEIPPSLGNLKKMHTLFLQVNRLTGRIPSELSGLESLMS 293 PTEFGSISTLKLLDLANCNLDGEIPPSLGNLKKLHSLFLHANRLTGHIPSELSGLESLMS 296 PPEFGSITTLKLLDLGSCNLDGEIPASLGNLKKLHSLFLQMNRLTGYIPPELSGLESLMS 290 $* * * * .: * * * * * * . . * * *: * *: * * * . * * * *: *: * * *: * * * * * * * * * * * * * * * *$ b Solyc04g081590.2 (SICLV1) AT1G75820

GSMUA_Achr3G26680_001

Bradī̄ 30160

Brast07G235600

LOC_Os06g50340

Sevir.4G294000

Seita.4G281800

Pahal.D00165

Pavir.Db00153

Sobic.010G267700

GRMZM2G300133

MDP 0000280399

AHYPO 018678

Migut.N03171

Migut.C00856

DCAR 022991

PGSC0003DMG 400009941

Eucgr.H00964

Eucgr.H00963

Phvul.011G042000

Glyma.11G114100

Glyma.12G040000

Aqcoe6G222600

Prupe.6G163000

Kaladp0068s0368

Kalax.0183s0036

Bol027692

Brara.G03381

Araha.9358s0001

AL2G35810

Thhalv10018069m.g

Bostr.20129s0016

Cagra.0799s0053

Carubv10019714m.g

MDP 0000804929

gene08548-v1.0-hybrid

Prupe.1G363300

GSVIVG01009941001

Potri.002G019900

SapurV1A.0025s0150

Potri.005G241500

SapurV1A.0384s0100

Manes.05G145600

30170.t000788

evm.TU.supercontig_26.309

Ciclev10000156m.g

orange $1.1 \mathrm{~g} 002010 \mathrm{~m} . \mathrm{g}$

Gorai.005G112100

Thecc1EG034252

Medtr4g070970

Tp57577_TGAC_v2_gene30515

Lus $10040592 . \bar{g}$
EELRLGYYNSYEGGIPSE-FGNISTLKLLDLGNCNLDGEVPPSLGNLKKLHSLFLOVNRI REMYIGYYNSYTGGVPPE-FGGLTKLEILDMASCTLTGE IPTSLSNLKHLHTLFLH INNI --------YEGGIPWE-FGRLSSLVRLDMAGCRLSGTLPASLGQLKRLDSLFLQINRI EDLYLGYFNQYDGGVPPE-FGELASLVRLDMSSCNLTGPVPPELGKLSKLQTLFLLWNRL QDLYLGYYNQYDGGVPPE-FGALGSLIRLDMSSCNLTGP IPPELGMLSNLETLFLQWNRL REMYIGYYNQYDGGVPPE-FGDLGALLRLDMSSCNLTGPVPPELGRLQRLDTLFLQWNRI REMYIGYYNOYTGGVPPE-FGDLRSLVRLDISSCNLTGPVPPELARLTOLDTLFLS INOL REMY IGYYNOYTGGVPPE-FGDLRSLVRLDISSCNLTGPVPPELARLTOLDTLFLS INOI REMYIGYFNQYTGGVPPE-FGDLRSLVRLDMSSCNLTGPVPLELARLTQLDTLFLSINQL REMYIGYFNOYTGGVPPE-FGDLRSLVRLDMSSCNLTGPVPPELARLTOLDTLFLSINOL REMYVGYYNQYSGGVPPE-FGDLQSLVRLDMSSCTLTGPIPPELARLSRLDTLFLSMNQL REMYVGYYNQYSGGVPRE-FGALQSLVRLDMSSCTLTGPIPPELARLSRLDTLFLALNQL KELYLGYYNNYDGGIPPE-FGSLPLLKVLDMSSCNLIGKIPTSLSLLKNLHSLFLOVNRI NMLFLGYYNTFSGGIPSE-FGSLSSLKLLDMASCNLSGE I PKTLGNLKNVHTLFLQRNQL QELYLGYFNTYDGGIPPA-FGSISTLQLLDLAMCNLTGE IPASLGNLKHLHSLFLQVNNL LELYLGYYNTYSGGIPPE-FGS ISSLQLLDLGMCNLTGEIPATLGNLKHLHTLFLQVNNL QILRLGYYNMYLGGIPSE-LGTLSDLRLLDLGGCNLTGE IPASLGNLKLLHTLFLQYNNL EELRLGYYNSYEGGIPSE-FGNISTLKLLDLGNCNLDGEVPPSLGNLKKLHTLFLQVNRL RGLYLGYFNAFDGGIPAE-FGSLKELRILDMASCGLSGEIPASLGELKKLDSLFLHLNKI QWLYLGYFNTYDGEIPAE-FGSMKELRRLDLASCGLSGE IPVSLSELKKLDSLFLQWNNL KYLKLGYNNAYEGGIPPE-FGAMKSLIYLDLSSCNLSGEIPPSLSSLKKLDTLFLQMNNL RILKLGYNNAYEGGIPPE-FGTMESLKYLDLSSCNLSGE IPPSLANMRNLDTLFLQMNNL RYLKLGYNNAYEGGIPPE-FGSMKSLRYLDLSSCNLSGE IPPSLANLTNLDTLFLQINNL QQLYLGYYNAYEGGIPPE-FGSFESLRLLDLGSCNLSGEIPASLGGLKLLDTLFLQFNHL KELYVGYFNSFDGGIPPE-LGSLTWLOVLDLASCNLSGS IPRSLGLLKHLRSLFLOVNCL EQMYVGYFNVYSSGIPPE-FGSI ISLRILDMASCNLSGE IPATLGKLKNLDTLFLQVNNF EQMYVGYFNVYSSGIPPE-FGSITSLRILDMASCNLSGE IPATLGKLKNLDTLFLQVNNF KEMYVGYFNSYTGGVPPE-FGELTNLEVLDMASCTLTGE IPTTLSNLKHLHTLFLH INNL KEMYVGYFNSYTGGVPPE-FGELSNLEVLDMASCTLTGE IPTTLSNLKHLHTLFLH INNI KEMY IGYYNSYTGGVPPE-FGGLTKLE ILDMASCTLTGE IPTSLSNLKHLHTLFLH INNL KEMYIGYYNSYTGGIPPE-FGGLTKLE ILDMASCTLTGE IPTSLSNLKHLHTLFLHVNNL REMYVGYYNSYTGGVPPE-FGGLTKLEVLDMASCTLTGE IPTTLSNLKHLHTLFLHINNL REMYVGYYNSYTGGVPPE-FGGLTKLEILDMASCTLTGE IPTSLSNLKHLNTLFLH INNL REMYVGYFNSYTGGVPPE-FGGLTKLQILDMASCTLTGEIPTSLSNLKHLHTLFLHINNL REMYVGYFNSYTGGVPPE-FGGLTKLQILDMASCTLTGEIPTSLSNLKHLHTLFLHINNL RELYVGYYNSYSGGIPPE-LGSLSSLQILDMGSCNLVGP IPTTLSLLKHLHTLFLQVNRI KEMYVGYFNSYDGGIPPE-LGSLSSLRVLDMASCNLTGTIPISLSNLKHLHSLFLQINQL KEMYVGYFNSYDGGIPPE-LGSLSSLQVLDMASCNLSGTIPTNLSLLKNLNSLFLQVNRI OGLFLGYFNIYEGGIPPE-LGLLSSLRVLDLGSCNLTGE IPPSLGRLKMLHSLFLQLNOI KSLCIGYYNHYEGGIPPE-FGSLSNLELLDMGSCNLNGE IPSTLGQLTHLHSLFLQFNNL KSLSIGYFNHYEGGIPPE-FGSLSSLELLDMGSCNLNGE IPSTLGQLTRLQSLFLQFNNL KSLCVGYFNRYEGSIPPE-FGSLSNLELLDMASCNLDGE IPSALSQLTHLHSLFLQVNNL KSLCVGYFNHYEGI IPPE-FGSLVNLELLDMASCNLNGE IPATLGOLTRLHSLFLOVNNL RSLYLGYYSSYEGGIPPE-FGFLSSLEVLDMAFCNLTGEIPSTLGLLKRLHTLFLQMNNL RKLYLGYFNSWEGGIPPE-FGSLSSLE ILDMAQSNLSGE IPPSLGQLKNLNSLFLQMNRL KALF IGYSNLYNGGVPRE-FGDLSELOILDMSSCNITGE IPTSLSNLKHLHSLFLOMNNI REMYIGYFNTYTGGISPE-FGALTQLQVLDMASCNISGEIPTSLSRLKLLHSLFLQMNKL REMY IGYFNTYTGGIPPG-FGALTOLOVLDMASCNISGE IPTSLSRLKLLHSLFLOMNKI KYLVIGYFNAYDGGIPPE-YGSLSQLELLDMASCNITGE IPSSLSNLKHLHSLFLQLNRI KEMYIGYFNAYVGE IPPE-FGTLSQLQVLDMASCNLTGE IPVSLSNLKHLHTLFLQLNRL KELOLGYENAYSGGIPPE-LGS IKSLRYLE ISNANLTGE IPPSLGNLENLDSLFLOMNNL KELRLGYNNAYEGGVPPE-FGSMKYLRYLEMPSCNLTGE IPPSLGNLENLDSLFLQGNNL KELYLGYFNSFSGGIPSGMFOGLTSLRVLDMASCNLSGE IPPSLGQLKNLRACYLQLNHF

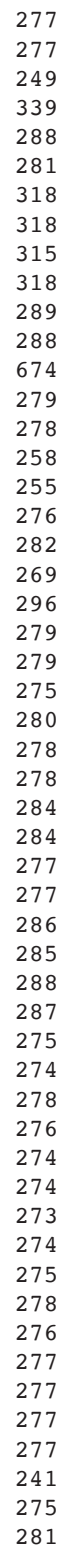

\section{Supplementary Fig. 4. Sequence alignments of CLV1 receptor homologs.}

a, Alignment of the Solanaceae CLV1 protein sequences. Red letters indicate the two ultra-conserved amino acids involved in the physical binding of CLE dodecapeptides. b, Alignment of CLV1 homologs in angiosperms. All the sequences are from the Phytozome v12.1 database (phytozome.jgi.doe.gov). Yellow highlights mark the conserved Asp and Phe. Detailed sequence information is shown in Supplementary Dataset 3. 
bioRxiv preprint doi: https://doi.org/10.1101/2022.01.03.474791; this version posted January 3, 2022. The copyright holder for this preprint (which was not certified by peer review) is the author/funder. All rights reserved. No reuse allowed without permission.

Supplementary Fig. 5

a

PhCLV1

(Peaxi162Scf00553g00620)

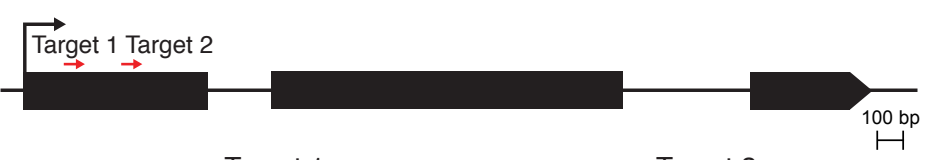

Target 1

Target 2

WT ACACTTATGAAACTTAAAGAATCCATGGTT (115) TATTTGGTACCATACCACCAGAAATTGGTT phclv $1^{\text {CR-1 }}$ ACACTTATGAAACTTAAAGAATTCCATGGTT (115) TATTTGGTACCATACCACCAGAAATTGGTT

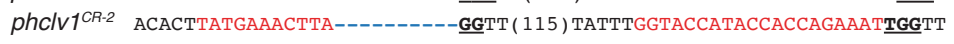

b

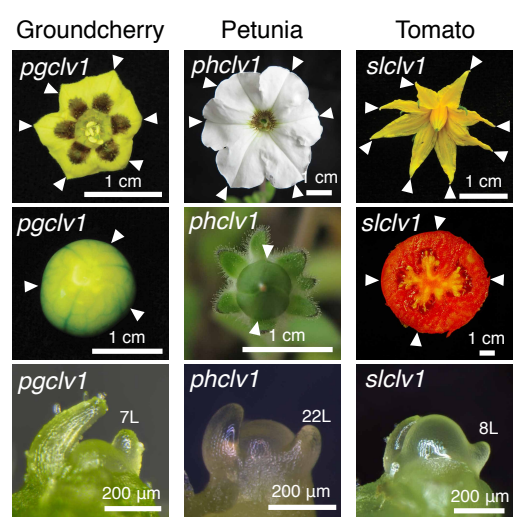

C
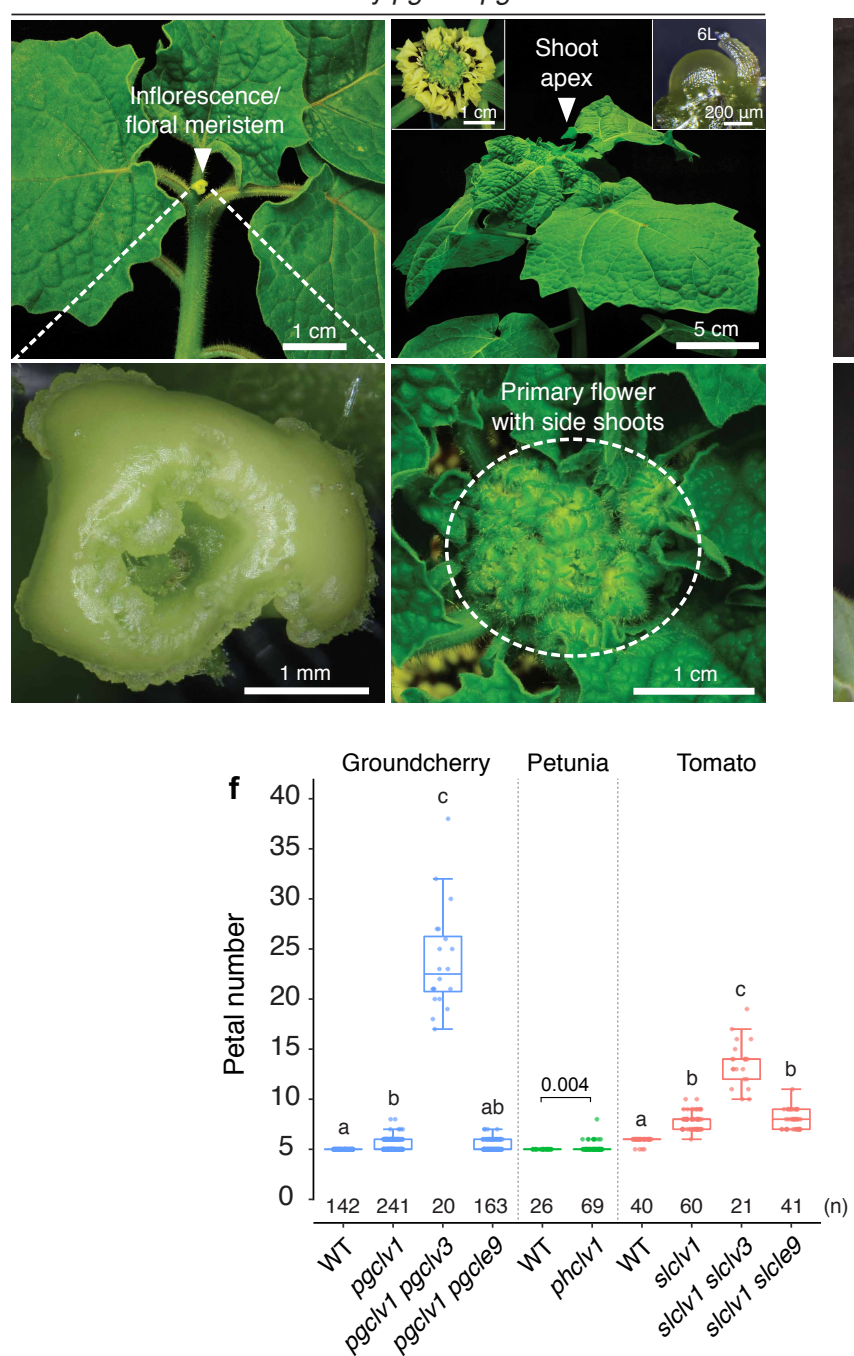

\section{d Petunia pholv1 pholv3}

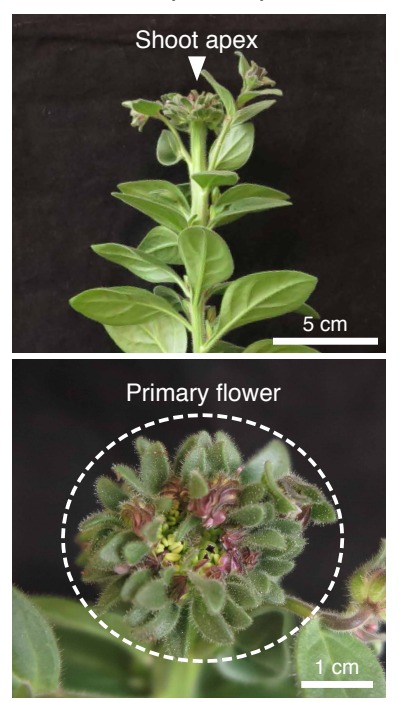

e Tomato slclv1 slclv3
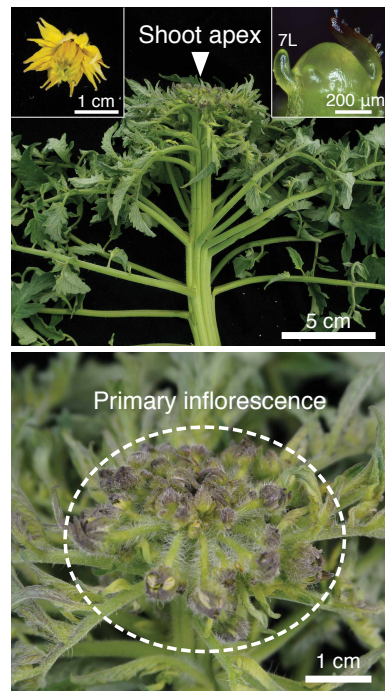

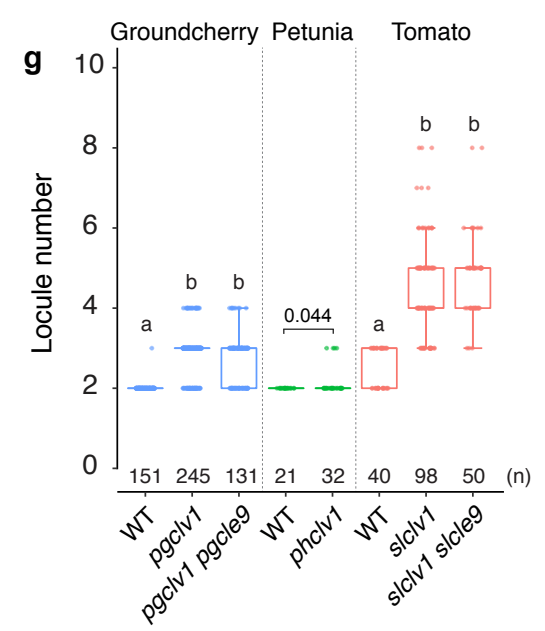

Supplementary Fig. 5. Groundcherry pgclv1 pgclv3 and petunia phclv1 phclv3 double mutants are severely fasciated like tomato slclv1 slclv3 double mutants.

a, Gene structure and sequences of two phclv1 CRISPR mutants. Guide RNA and PAM sequences are highlighted in red and bold underlined, respectively. Blue letters and dashes indicate insertions and deletions, respectively. Numbers in parentheses represent gap lengths. b, Flowers, fruits/pods, and meristems from $p g c l v 1$, phclv1, and slclv1 single mutants. White arrowheads mark petals or locules. $7 \mathrm{~L}$, 7 th 1 eaf primordium, $8 \mathrm{~L}, 8^{\text {th }} 1$ leaf primordium. 22L, 22th leaf primordium. C, Side and top-down views of a pgclv1 pgclv3 double mutant shoot, inflorescence/floral meristem, and primary inflorescence. $6 \mathrm{~L}, 6^{\text {th }}$ leaf primordium. D, Side and top-down views of a phclv1 phclv3 double mutant shoot and primary flower. E, Side and top-down views of a slclv1 slclv3 double mutant shoot, flower, vegetative meristem and primary inflorescence. Fasciated flowers and vegetative meristems are shown in insets of $\mathbf{c}$ and e. f, g, Petal (f) and locule (g) numbers of groundcherry WT, pgclv1, pgclv1 pgclv3, pgclv1 pgcle9, and petunia WT, phclv1, and tomato WT, slclv1, slclv1 slclv3, and slclv1 slcle9. Not that all three Solanaceae $c l v 1$ single mutant fasciation phenotypes are similarly weak. Box plots, $25^{\text {th }}-75^{\text {th }}$ percentile; center line, median; whiskers, full data range in $\mathbf{d}$ and $\mathbf{e}$. The letters indicate the significance groups at $P<0.01$ (One-way ANOVA and Tukey test) in $\mathbf{f}$ and $\mathbf{g}$. $P$ values (two-tailed, two-sample $t$-test) in $\mathbf{f}$ and $\mathbf{g}$. Exact sample sizes (n) are shown in $\mathbf{f}$ and $\mathbf{g}$. 


\section{Supplementary Fig. 6}

a
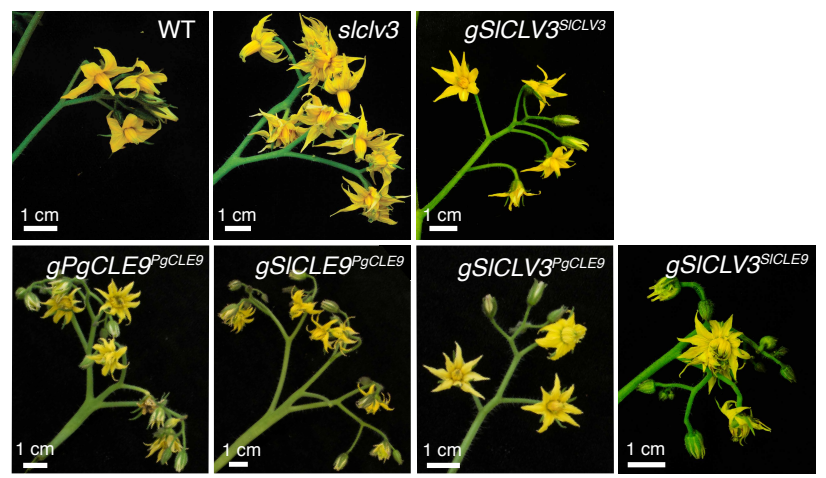

C
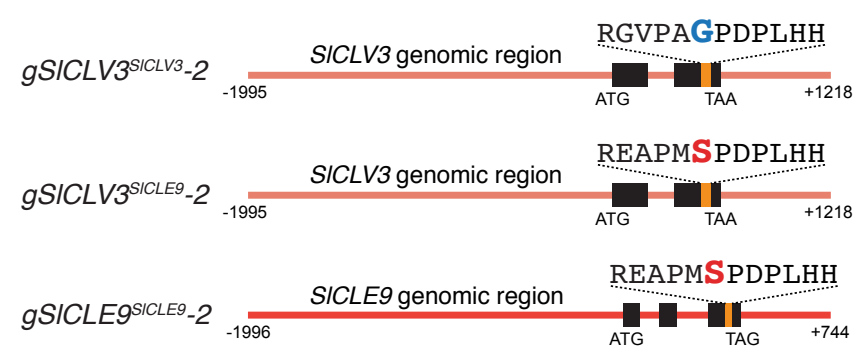

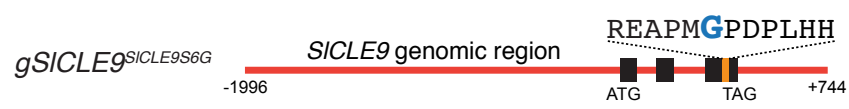

b

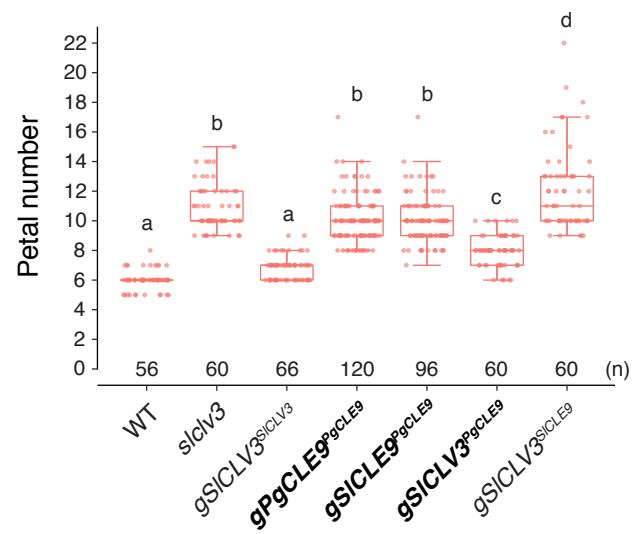

d

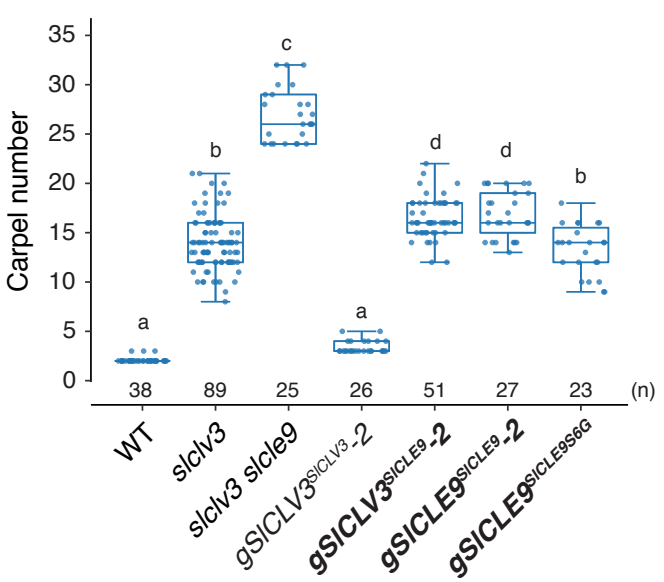

\section{Supplementary Fig. 6. Transgenic complementation tests of tomato slclv3 single and slclv3 slcle9 double mutants.}

a, b, Complementation tests of tomato slclv3 single mutants. Inflorescence images (a) and petal number quantifications (b) of WT and slclv3 compared to the $\mathrm{T}_{1}$ transgenic plants $g S l C L V 3$ SICLV3, gPgCLE9PgCLE9, gSlCLE9PgCLE9, gSlCLV3PgCLE9, and gSlCLV3SICLE9. c, Diagrams of the constructs used for complementation tests of slclv3 slcle9 double mutants. gSlCLV3SlCLV3 (SlCLV3 genomic DNA). gSlCLV3SICLE9 (SlCLV3 genomic DNA including the sequence for SICLE9 dodecapeptide). gSlCLE9SICLE9 (SlCLE9 genomic DNA). gSlCLE9SICLE9S6G (SlCLE9 genomic DNA including the sequence for PgCLE9 dodecapeptide). Black and orange rectangles mark the coding sequences and the dodecapeptide sequences, respectively. The numbers with minus (-) and plus (+) signs indicate the positions of the upstream sequences and the downstream sequences from the adenines of start codons, respectively. d, Carpel number quantifications of WT, slclv3, slclv3 slcle9 mutants compared to the $\mathrm{T}_{1}$ transgenic plants gSlCLV3SlCLV3-2, gSICLV3SICLE9-2, gSICLE9SICLE9-2, and gSICLE9 SICLE9S6G. Data are based on at least three independent transgenic lines for each construct. Box plots, $25^{\text {th }}-75^{\text {th }}$ percentile; center line, median; whiskers, full data range in $\mathbf{b}$ and $\mathbf{d}$. The letters indicate the significance groups at $P<0.01$ (One-way ANOVA and Tukey test) in $\mathbf{b}$ and $\mathbf{d}$. Exact sample sizes (n) are shown in $\mathbf{b}$ and $\mathbf{d}$. 